\title{
Monotone Iterative Schemes for Positive Solutions of a Fractional $q$-Difference Equation with Integral Boundary Conditions on the Half-Line
}

\author{
Min Jiang $\mathbb{B D}^{1}$ and Rengang Huang $\mathbb{B D}^{2}$ \\ ${ }^{1}$ School of Data-Science and Information-Engineering, Guizhou Minzu University, Guiyang 550025, China \\ ${ }^{2}$ School of Business, Guizhou Minzu University, Guiyang 550025, China \\ Correspondence should be addressed to Min Jiang; minjiang0701@163.com
}

Received 19 August 2020; Revised 25 December 2020; Accepted 29 January 2021; Published 26 February 2021

Academic Editor: Ghulam Shabbir

Copyright (C) 2021 Min Jiang and Rengang Huang. This is an open access article distributed under the Creative Commons Attribution License, which permits unrestricted use, distribution, and reproduction in any medium, provided the original work is properly cited.

In this paper, we study the boundary value problem of a fractional $q$-difference equation with nonlocal integral boundary conditions on the half-line. Using the properties of the Green function and monotone iterative method, the extremal solutions are obtained. Finally, an example is presented to illustrate our main results.

\section{Introduction}

In this paper, we are concerned with the following fractional $q$-differential equation with integral boundary value problem on the half-line:

$$
\left\{\begin{array}{l}
D_{q}^{\alpha} u(t)+f\left(t, u(t), D_{q}^{\alpha-1} u(t), D_{q}^{\alpha-2} u(t), \ldots, D_{q}^{\alpha-(n-1)} u(t)\right)=0, \quad n-1<\alpha \leq n \\
u(0)=D_{q}^{j} u(0)=0, \quad j=1, \ldots, n-2, \\
D_{q}^{\alpha-1} u(+\infty)=\int_{0}^{+\infty} g(t) u(t) \mathrm{d}_{q} t
\end{array}\right.
$$

where $t \in J=[0,+\infty), D_{q}^{\alpha}$ and $D_{q}^{\alpha-j}$ are the fractional $q$-derivative of Riemann-Liouville type of order $\alpha$ and $\alpha-j$, $D_{q}^{j} \quad$ is the $q$-derivative of order $j$, $0<q<1, n \in \mathbb{N}, n \geq 3, f \in C\left(J \times \mathbb{R}^{n}, J\right)$, and $g$ is a given function and satisfies some conditions which will be given later.

The $q$-difference calculus was initially developed by Jackson $[1,2]$ and had proved to have important applications in many subjects, such as quantum mechanics, complex analysis, and hypergeometric series. The fractional $q$-difference calculus had its origin in the works by Al-Salam [3] and Agarwal [4]. Due to the development of the fractional differential equations, fractional $q$-differential equations, regarded as fractional analogue of $q$-difference equations, have been studied by several researchers, especially, about the existence of the solutions for the boundary value problems (see [5-22] and the references therein). 
In [21], Yang studied the coupled integral boundary value problem for systems of nonlinear fractional $q$-difference equations as follows:

$$
\left\{\begin{array}{l}
D_{q}^{\alpha} u(t)+\lambda f(t, u(t), v(t))=0, \\
D_{q}^{\beta} v(t)+\lambda g(t, u(t), v(t))=0, \quad t \in(0,1), \lambda>0 \\
D_{q}^{j} u(0)=D_{q}^{j} v(0)=0, \quad 0 \leq j \leq n-2, \\
u(1)=\mu \int_{0}^{1} v(s) \mathrm{d}_{q} s \\
v(1)=v \int_{0}^{1} u(s) \mathrm{d}_{q} s
\end{array}\right.
$$

where $\lambda, \mu$, and $\nu$ are three parameters with $0<\mu<[\beta]_{q}$ and $0<\nu<[\alpha]_{q}, \alpha, \beta \in(n-1, n]$ are two real numbers and $n \geq 3$, and $D_{q}^{\alpha}$ and $D_{q}^{\beta}$ are the fractional $q$-derivative of Riemann-Liouville type. By applying the nonlinear alternative of Leray-Schauder type and Krasnoselskii's fixed point theorems, the authors obtain some sufficient conditions for the existence of positive solutions to the considered problem.

In [14], Wang et al. investigated the following nonlinear fractional $q$-difference equation with $q$-integral boundary condition:

$$
\left\{\begin{array}{l}
D_{q}^{\alpha} u(t)+f\left(u(t),\left(I_{q}^{\beta} u\right)(t)\right)=0, \quad t \in(0,1) \\
D_{q}^{j} u(0)=0, \quad 0 \leq j \leq n-2, \\
u(1)=\mu \int_{0}^{1} u(s) \mathrm{d}_{q} s
\end{array}\right.
$$

where $\alpha \in(n-1, n], n \geq 3, \mu$ is a parameter with $0<\mu<[\alpha]_{q}$, $\beta>0$, and $D_{q}^{\alpha}$ is the fractional $q$-derivative of Riemann-Liouville type. By applying the hybrid monotone method, the existence and uniqueness of the positive solution of the $q$-integral boundary problem are obtained.

In [17], the authors investigate the existence of solutions for the following boundary value problem of nonlinear fractional $q$-difference equations on the half-line

$$
\left\{\begin{array}{l}
D_{q}^{\alpha} u(t)+f(t, u(t))=0 \\
u(0)=0 \\
D_{q}^{\alpha-1} u(+\infty)=\sum_{i=1}^{m} a_{i} u\left(\xi_{i}\right)
\end{array}\right.
$$

where $1<\alpha<2,0 \leq \sum_{i=1}^{m} a_{i} \xi_{i}^{\alpha-1}<\Gamma_{q}(\alpha) . D_{q}^{\alpha}$ is the $q$-derivative of Riemann-Liouville type of order $\alpha$. By means of Schauder fixed point theorem and Leggett-Williams fixed point theorem, some results on existence and multiplicity of solutions to the above boundary value problem are obtained.

To the best of our knowledge, there are few papers that consider the boundary value of nonlinear fractional $q$-difference equations with nonlocal conditions on the half-line, although the study of such problems is very important. In [17], the authors only proved the existence and multiplicity of solutions, for the uniqueness of positive solutions without being given. Moreover, how to seek the solutions? It is very important and helpful for computational purpose. This thought motivates the research of iterative schemes of positive solutions for problem (1). We should mention that the paper has some new features. Firstly, we consider the infinite interval problem for higher-order nonlinear fractional $q$-difference equation with integral boundary conditions. Secondly, the nonlinearity relies on the lower-order fractional $q$-derivative of unknown function. Our main purpose of this paper is by constructing a suitable Banach space, defining appropriate operators, and using the monotone iterative method, which is different from the method in [17] to obtain the existence and uniqueness of positive solutions.

\section{Preliminaries on $q$-Calculus and Lemmas}

Here we recall some definitions and fundamental results on fractional $q$-integral and fractional $q$-derivative. For more information regarding fractional $q$-calculus, see [23].

Let $q \in(0,1)$ and define

$$
[a]_{q}=\frac{1-q^{a}}{1-q}, \quad a \in \mathbb{R} .
$$

The $q$-analogue of the power function $(a-b)^{(n)}$ with $n \in N_{0}$ is

$$
\begin{aligned}
& (a-b)^{(0)}=1 \\
& (a-b)^{(n)}=\prod_{k=0}^{n-1}\left(a-b q^{k}\right), \quad n \in N, a, b \in \mathbb{R} .
\end{aligned}
$$

More generally, if $\alpha \in R$, then

$$
(a-b)^{(\alpha)}=a^{\alpha} \prod_{n=0}^{\infty} \frac{a-b q^{n}}{a-b q^{\alpha+n}} .
$$
by

If $b=0$, then $a^{(\alpha)}=a^{\alpha}$. The $q$-gamma function is defined

$$
\Gamma_{q}(s)=\frac{(1-q)^{(s-1)}}{(1-q)^{s-1}}, \quad s \in R,\{0,-1,-2, \ldots\}
$$

and satisfies $\Gamma_{q}(s+1)=[s]_{q} \Gamma_{q}(s)$. The $q$-derivative of a function $u$ is defined by

$$
\begin{aligned}
& \left(D_{q} u\right)(s)=\frac{u(s)-u(q s)}{(1-q) s}, \\
& \left(D_{q} u\right)(0)=\lim _{s \longrightarrow 0}\left(D_{q} u\right)(s),
\end{aligned}
$$

and $q$-derivative of higher order by

$$
\left(D_{q}^{0} u\right)(s)=u(s) \text { and }\left(D_{q}^{n} u\right)(s)=D_{q}\left(D_{q}^{n-1} u\right)(s), \quad n \in N \text {. }
$$

The $q$-integral of a function $f$ defined in the interval $[0, b]$ is given by

$$
\left(I_{q} u\right)(s)=\int_{0}^{s} u(t) \mathrm{d}_{q} t=s(1-q) \sum_{n=0}^{\infty} u\left(s q^{n}\right) q^{n}, \quad s \in[0, b] .
$$

If $a \in[0, b]$ and $u$ is defined in the interval $[0, b]$, its integral from $a$ to $b$ is defined by 


$$
\int_{a}^{b} u(t) \mathrm{d}_{q} t=\int_{0}^{b} u(t) \mathrm{d}_{q} t-\int_{0}^{a} u(t) \mathrm{d}_{q} t
$$

Similar to that for derivatives, an operator $I_{q}^{n}$ can be defined, namely,

$$
\left(I_{q}^{0} u\right)(s)=u(s) \text { and }\left(I_{q}^{n} u\right)(s)=I_{q}\left(I_{q}^{n-1} u\right)(s), \quad n \in N
$$

The fundamental theorem of calculus applies to these operators $I_{q}$ and $D_{q}$, i.e.,

$$
\left(D_{q} I_{q} u\right)(s)=u(s),
$$

and if $u$ is continuous at $s=0$, then

$$
\left(I_{q} D_{q} u\right)(s)=u(s)-u(0) .
$$

The basic properties of the two operators can be found in the book [23].

The following definition was considered first in [4].

Definition 1 (see [4]). Let $\alpha \geq 0$ and $u$ be a function defined on $[0,1]$. The fractional $q$-integral of the Riemann-Liouville type is $\left(I_{q}^{0} u\right)(s)=u(s)$ and

$$
\left(I_{q}^{\alpha} u\right)(s)=\frac{1}{\Gamma_{q}(\alpha)} \int_{0}^{s}(s-q t)^{(\alpha-1)} u(t) \mathrm{d}_{q} t, \quad \alpha>0, s \in[0,1] .
$$

Definition 2 (see [24]). The fractional $q$-derivative of the Riemann-Liouville type of order $\alpha \geq 0$ is defined by $\left(D_{q}^{0} u(s)=u(s)\right)$ and

$$
\left(D_{q}^{\alpha} u\right)(s)=\left(D_{q}^{m} I_{q}^{m-\alpha} u\right)(s), \quad \alpha>0,
$$

where $m$ is the smallest integer greater than or equal to $\alpha$.

Lemma 1 (see $[4,24])$. Let $\alpha, \beta \geq 0$ and $u$ be a function defined on $[0,1]$. Then, the following formulas hold:

$$
\begin{aligned}
& \text { (1) }\left(I_{q}^{\beta} I_{q}^{\alpha} u\right)(s)=\left(I_{q}^{\alpha+\beta} u\right)(s) \\
& \text { (2) }\left(D_{q}^{\alpha} I_{q}^{\alpha} u\right)(s)=u(s)
\end{aligned}
$$

Lemma 2 (see [25]). Let $\alpha>0$ and $n$ be a positive integer. Then, the following equality holds:

$$
\left(I_{q}^{\alpha} D_{q}^{n} u\right)(s)=\left(D_{q}^{n} I_{q}^{\alpha} u\right)(s)-\sum_{k=0}^{n-1} \frac{s^{\alpha-n+k}}{\Gamma_{q}(\alpha+k-n+1)}\left(D_{q}^{k} u\right)(0) .
$$

Lemma 3 (see [24]). For $\beta \in \mathbb{R}^{+}, \lambda \in(-1, \infty)$, the following is valid:

$$
I_{q}^{\beta}\left((s-a)^{(\lambda)}\right)=\frac{\Gamma_{q}(\lambda+1)}{\Gamma_{q}(\beta+\lambda+1)}(s-a)^{(\beta+\lambda)}, \quad 0<a<x<b .
$$

In the following, we introduce the hypotheses that will be used in subsequent proof:

$\left(\mathrm{H}_{1}\right): g(t)$ satisfies $\int_{0}^{+\infty} t^{\alpha-1} g(t) \mathrm{d}_{q} t=\Lambda<\Gamma_{q}(\alpha)$ and $f(t, 0, \ldots, 0) \equiv 0, \forall t \in J=[0,+\infty)$.

(H2): there exist nonnegative functions $a_{0}(t), a_{i}(t) \in L_{q}[0,+\infty)$ and constants $\lambda_{i} \geq 0$ satisfying

$$
\begin{aligned}
& \left|f\left(t, u_{1}, u_{2}, \ldots, u_{n}\right)\right| \leq a_{0}(t)+\sum_{i=1}^{n} a_{i}(t)\left|u_{i}\right|^{\lambda_{i}}, \quad \forall t \in J, u \in \mathbb{R}, i=1,2, \ldots, n \\
& \int_{0}^{+\infty} a_{0}(t) \mathrm{d}_{q} t=a_{0}<+\infty \\
& \int_{0}^{+\infty}\left(1+t^{\alpha-1}\right)^{\lambda_{1}} a_{1}(t) \mathrm{d}_{q} t=a_{1}<+\infty \\
& \int_{0}^{+\infty} a_{2}(t) \mathrm{d}_{q} t<+\infty \\
& \int_{0}^{+\infty}\left(1+t^{i-2}\right)^{\lambda_{i}} a_{i}(t) \mathrm{d}_{q} t=a_{i}<+\infty, \quad i=3, \ldots, n .
\end{aligned}
$$


$\left(\mathrm{H}_{3}\right)$ : there exist nonnegative functions

$b_{i}(t) \in L_{q}[0,+\infty)$ satisfying

$$
\begin{aligned}
& \left|f\left(t, u_{1}, u_{2}, \ldots, u_{n}\right)-f\left(t, v_{1}, v_{2}, \ldots, v_{n}\right)\right| \leq \sum_{i=1}^{n} b_{i}(t)\left|u_{i}-v_{i}\right|, \quad \forall t \in J, u_{i}, v_{i} \in \mathbb{R}, i=1,2, \ldots, n \\
& \int_{0}^{+\infty}\left(1+t^{\alpha-1}\right) b_{1}(t) \mathrm{d}_{q} t=b_{1}<+\infty \\
& \int_{0}^{+\infty}\left(1+t^{i-2}\right) b_{i}(t) \mathrm{d}_{q} t=b_{i}<+\infty, \quad i=3, \ldots, n \\
& \int_{0}^{+\infty} b_{2}(t) \mathrm{d}_{q} t<+\infty \\
& \int_{0}^{+\infty}|f(t, 0,0, \ldots, 0)| \mathrm{d}_{q} t=\kappa<+\infty .
\end{aligned}
$$

$\left(\mathrm{H}_{4}\right)$ : function $f\left(t, u_{1}, u_{2}, \ldots, u_{n}\right)$ is increasing with respect to the variables $u_{1}, u_{2}, \ldots, u_{n}, \forall t \in J$.

Lemma 4. Assume that $g(t)$ satisfies condition $\left(H_{1}\right)$, then fractional $q$-differential equation

$$
\left\{\begin{array}{l}
D_{q}^{\alpha} u(t)+h(t)=0, \quad n-1<\alpha \leq n, \\
u(0)=D_{q}^{j} u(0)=0, \quad j=1, \ldots, n-2, \\
D_{q}^{\alpha-1} u(+\infty)=\int_{0}^{+\infty} g(t) u(t) \mathrm{d}_{q} t,
\end{array}\right.
$$

has a unique integral representation

$$
u(t)=\int_{0}^{+\infty} G(t, q s) h(s) \mathrm{d}_{q} s
$$

where

$$
\begin{aligned}
G(t, q s) & =G_{1}(t, q s)+G_{2}(t, q s), \\
G_{1}(t, q s) & =\frac{1}{\Gamma_{q}(\alpha)} \begin{cases}t^{\alpha-1}-(t-q s)^{(\alpha-1)}, & 0 \leq s \leq t \leq+\infty, \\
t^{\alpha-1}, & 0 \leq t \leq s \leq+\infty,\end{cases} \\
G_{2}(t, q s) & =\frac{t^{\alpha-1}}{\Gamma_{q}(\alpha)-\Lambda} \int_{0}^{+\infty} g(t) G_{1}(t, q s) \mathrm{d}_{q} t .
\end{aligned}
$$

Proof. In view of Definition 2 and Lemma 1, we see that

$$
I_{q}^{\alpha} D_{q}^{n} I_{q}^{n-\alpha} u(t)=-I_{q}^{\alpha} h(t)
$$

By Lemma 2, we obtain

$$
u(t)=-I_{q}^{\alpha} h(t)+c_{1} t^{\alpha-1}+c_{2} t^{\alpha-2}+\cdots+c_{n} t^{\alpha-n},
$$

where $c_{i}, i=1,2, \ldots, n$ are some constants. Since $u(0)=D_{q}^{j} u(0)=0, j=1, \ldots, n-2$, we get

$$
c_{2}=c_{3}=\cdots=c_{n}=0 \text {, }
$$

which implies that

$$
u(t)=-\frac{1}{\Gamma_{a}(\alpha)} \int_{0}^{t}(t-q s)^{(\alpha-1)} h(s) \mathrm{d}_{q} s+c_{1} t^{\alpha-1} .
$$

From (29) and Lemma 1, we have

$$
D_{q}^{\alpha-1} u(t)=-\int_{0}^{t} h(s) \mathrm{d}_{q} s+c_{1} \Gamma_{q}(\alpha) .
$$

Hence,

$$
D_{q}^{\alpha-1} u(+\infty)=-\int_{0}^{+\infty} h(s) \mathrm{d}_{q} s+c_{1} \Gamma_{q}(\alpha)
$$

The condition $D_{q}^{\alpha-1} u(+\infty)=\int_{0}^{+\infty} g(t) u(t) \mathrm{d}_{q} t$ together with (31) implies that

$$
c_{1}=\frac{1}{\Gamma_{q}(\alpha)}\left(\int_{0}^{+\infty} h(s) \mathrm{d}_{q} s+\int_{0}^{+\infty} g(t) u(t) \mathrm{d}_{q} t\right) .
$$

Submitting (32) to (29), we get

$$
\begin{aligned}
u(t) & =-\frac{1}{\Gamma_{q}(\alpha)} \int_{0}^{t}(t-q s)^{(\alpha-1)} h(s) \mathrm{d}_{q} s+\frac{t^{\alpha-1}}{\Gamma_{q}(\alpha)}\left(\int_{0}^{+\infty} h(s) \mathrm{d}_{q} s+\int_{0}^{+\infty} g(t) u(t) \mathrm{d}_{q} t\right) \\
& =\frac{1}{\Gamma_{q}(\alpha)} \int_{0}^{+\infty} t^{\alpha-1} h(s) \mathrm{d}_{q} s-\frac{1}{\Gamma_{q}(\alpha)} \int_{0}^{t}(t-q s)^{(\alpha-1)} h(s) \mathrm{d}_{q} s+\frac{t^{\alpha-1}}{\Gamma_{q}(\alpha)} \int_{0}^{+\infty} g(t) u(t) \mathrm{d}_{q} t \\
& =\int_{0}^{+\infty} G_{1}(t, q s) h(s) \mathrm{d}_{q} s+\frac{t^{\alpha-1}}{\Gamma_{q}(\alpha)} \int_{0}^{+\infty} g(t) u(t) \mathrm{d}_{q} t .
\end{aligned}
$$


Equation (33) is multiplied by $g(t)$ and $q$-integrating from 0 to $+\infty$, and we obtain

$$
\int_{0}^{+\infty} g(t) u(t) \mathrm{d}_{q} t=\int_{0}^{+\infty} g(t)\left(\int_{0}^{+\infty} G_{1}(t, q s) h(s) \mathrm{d}_{q} s\right) d_{q} t+\frac{\Lambda}{\Gamma_{q}(\alpha)} \int_{0}^{+\infty} g(t) u(t) \mathrm{d}_{q} t
$$

From (34), we get

$$
\int_{0}^{+\infty} g(t) u(t) \mathrm{d}_{q} t=\frac{\Gamma_{q}(\alpha)}{\Gamma_{q}(\alpha)-\Lambda} \int_{0}^{+\infty} g(t)\left(\int_{0}^{+\infty} G_{1}(t, q s) h(s) \mathrm{d}_{q} s\right) \mathrm{d}_{q} t
$$

Combining (33) and (35), we have

$$
\begin{aligned}
u(t) & =\int_{0}^{+\infty} G_{1}(t, q s) h(s) \mathrm{d}_{q} s+\frac{t^{\alpha-1}}{\Gamma_{q}(\alpha)-\Lambda} \int_{0}^{+\infty} g(t)\left(\int_{0}^{+\infty} G_{1}(t, q s) h(s) \mathrm{d}_{q} s\right) \mathrm{d}_{q} t \\
& =\int_{0}^{+\infty} G_{1}(t, q s) h(s) \mathrm{d}_{q} s+\int_{0}^{+\infty} G_{2}(t, q s) h(s) \mathrm{d}_{q} s=\int_{0}^{+\infty} G(t, q s) h(s) \mathrm{d}_{q} s .
\end{aligned}
$$

The proof is completed.

Lemma 5. From Lemma 4, by direct calculation, we have

$$
D_{q}^{\alpha-i} u(t)=\int_{0}^{+\infty} G_{i}^{*}(t, q s) h(s) \mathrm{d}_{q} s, \quad i=1,2, \ldots, n-1,
$$

where

$$
\begin{array}{ll}
G_{i}^{*}(t, q s) & =G_{i 1}^{*}(t, q s)+G_{i 2}^{*}(t, q s), \\
G_{i 1}^{*}(t, q s)=\frac{1}{\Gamma_{q}(i)}\left\{\begin{array}{lr}
t^{i-1}-(t-q s)^{(i-1)}, & 0 \leq s \leq t \leq+\infty, \\
t^{i-1}, & 0 \leq t \leq s \leq+\infty,
\end{array}\right. \\
G_{i 2}^{*}(t, q s)=\frac{\Gamma_{q}(\alpha) t^{i-1}}{\Gamma_{q}(i)\left(\Gamma_{q}(\alpha)-\Lambda\right)} \int_{0}^{+\infty} g(t) G_{1}(t, q s) \mathrm{d}_{q} t .
\end{array}
$$

Lemma 6. Function $G(t, q s)$ defined in (24) satisfies the following conditions: $0 \leq G(t, q s) \leq\left(t^{\alpha-1} / \Gamma_{q}(\alpha)-\Lambda\right)$ and $0 \leq\left(G(t, q s) / 1+t^{\alpha-1}\right) \leq\left(1 / \Gamma_{q}(\alpha)-\Lambda\right)$ for all $0 \leq t, s \leq+\infty$.

Proof. From (24), it is obvious that, for all $0 \leq t, s \leq+\infty$,

$$
\begin{aligned}
& 0 \leq G_{1}(t, q s) \leq \frac{t^{\alpha-1}}{\Gamma_{q}(\alpha)} \\
& 0 \leq G_{2}(t, q s) \leq \frac{t^{\alpha-1}}{\Gamma_{q}(\alpha)-\Lambda} \int_{0}^{+\infty} g(t) \frac{t^{\alpha-1}}{\Gamma_{q}(\alpha)} \mathrm{d}_{q} s=\frac{\Lambda t^{\alpha-1}}{\Gamma_{q}(\alpha)\left(\Gamma_{q}(\alpha)-\Lambda\right)}
\end{aligned}
$$

The above two inequalities imply that 


$$
\begin{aligned}
& 0 \leq G(t, q s)=G_{1}(t, q s)+G_{2}(t, q s) \leq \frac{t^{\alpha-1}}{\Gamma_{q}(\alpha)}+\frac{\Lambda t^{\alpha-1}}{\Gamma_{q}(\alpha)\left(\Gamma_{q}(\alpha)-\Lambda\right)}=\frac{t^{\alpha-1}}{\Gamma_{q}(\alpha)-\Lambda} \\
& 0 \leq \frac{G(t, q s)}{1+t^{\alpha-1}} \leq \frac{t^{\alpha-1}}{\left(\Gamma_{q}(\alpha)-\Lambda\right)\left(1+t^{\alpha-1}\right)} \leq \frac{1}{\Gamma_{q}(\alpha)-\Lambda}
\end{aligned}
$$

The proof is completed.

Lemma 7. Function $G_{i}^{*}(t, q s)$ defined in Lemma 5 satisfies the following conditions:

$$
\begin{aligned}
& 0 \leq G_{i}^{*}(t, q s) \leq \frac{\Gamma_{q}(\alpha) t^{i-1}}{\Gamma_{q}(i)\left(\Gamma_{q}(\alpha)-\Lambda\right)}, \quad i=1,2, \ldots, n-1, \\
& 0 \leq \frac{G_{i}^{*}(t, q s)}{1+t^{i-1}} \leq \frac{\Gamma_{q}(\alpha)}{\Gamma_{q}(i)\left(\Gamma_{q}(\alpha)-\Lambda\right)}, \quad i=2, \ldots, n-1,
\end{aligned}
$$

for all $0 \leq t, s \leq+\infty$.

Proof. For $0 \leq t, s \leq+\infty$ and $i=1,2, \ldots, n-1$, by Remark 2 and Lemma 5 , it is easy to get

$$
\begin{aligned}
& 0 \leq G_{i 1}^{*}(t, q s) \leq \frac{t^{i-1}}{\Gamma_{q}(i)}, \\
& 0 \leq G_{i 2}^{*}(t, q s) \leq \frac{\Gamma_{q}(\alpha) t^{i-1}}{\Gamma_{q}(i)\left(\Gamma_{q}(\alpha)-\Lambda\right)} \int_{0}^{+\infty} g(t) \frac{t^{\alpha-1}}{\Gamma_{q}(\alpha)} \mathrm{d}_{q} s=\frac{t^{i-1} \Lambda}{\Gamma_{q}(i)\left(\Gamma_{q}(\alpha)-\Lambda\right)}
\end{aligned}
$$

This gives for $i=2, \ldots, n-1$,

$0 \leq G_{i}^{*}(t, q s) \leq \frac{t^{i-1}}{\Gamma_{q}(i)}+\frac{t^{i-1} \Lambda}{\Gamma_{q}(i)\left(\Gamma_{q}(\alpha)-\Lambda\right)}=\frac{\Gamma_{q}(\alpha) t^{i-1}}{\Gamma_{q}(i)\left(\Gamma_{q}(\alpha)-\Lambda\right)}$

$0 \leq \frac{G_{i}^{*}(t, q s)}{1+t^{i-1}} \leq \frac{\Gamma_{q}(\alpha)}{\Gamma_{q}(i)\left(\Gamma_{q}(\alpha)-\Lambda\right)}$
The proof is completed.

In the following, we will construct a suitable Banach space. Let

$$
X=\left\{u \mid u, D_{q}^{\alpha-1} u, D_{q}^{\alpha-i} u \in C[0,+\infty),\left\|\frac{u}{1+t^{\alpha-1}}\right\|_{\infty},\left\|D_{q}^{\alpha-1} u\right\|_{\infty}<\infty,\left\|\frac{D_{q}^{\alpha-i} u}{1+t^{i-1}}\right\|_{\infty}<\infty, \quad i=2, \ldots, n-1\right\}
$$

with the norm

$$
\|u\|=\max \left\{\left\|\frac{u}{1+t^{\alpha-1}}\right\|_{\infty},\left\|\frac{D_{q}^{\alpha-i} u}{1+t^{i-1}}\right\|_{\infty},\left\|D_{q}^{\alpha-1} u\right\|_{\infty}, \quad i=2, \ldots, n-1\right\},
$$

where $\|u\|_{\infty}=\sup _{t \in J}|u(t)|, J=[0,+\infty)$. 
Similar to the proof of Lemma 2.2 in [26], we can prove $X$ is a Banach space. Note that the Arzela-Ascoli theorem fails to work in $X$. In order to proceed, we need the following compactness criterion.

Lemma 8. Let $B \subset X$ be a bounded set. Then, $B$ is relatively compact in $X$ if the following conditions hold:

(i) $\left\{\left(u / 1+t^{\alpha-1}\right) \mid u \in B\right\}$, $\left\{\left(D_{q}^{\alpha-i} u / 1+t^{i-1}\right) \mid u \in B\right\},\left\{D_{q}^{\alpha-1} u \mid u \in B\right\}, i=$ $2, \ldots, n-1$ are equicontinuous on $[0, T]$ for any $T \in[0,+\infty)$

(ii) For $\forall \varepsilon>0, \exists T(\varepsilon)>0$ such that for any $t_{1}, t_{2} \geq T(\varepsilon), u \in B$, the following inequalities hold:

$$
\begin{aligned}
& \left|\frac{u\left(t_{2}\right)}{1+t_{2}^{\alpha-1}}-\frac{u\left(t_{1}\right)}{1+t_{1}^{\alpha-1}}\right|<\varepsilon, \\
& \left|\frac{D_{q}^{\alpha-i} u\left(t_{2}\right)}{1+t_{2}^{i-1}}-\frac{D_{q}^{\alpha-i} u\left(t_{1}\right)}{1+t_{1}^{i-1}}\right|<\varepsilon, \\
& \left|D_{q}^{\alpha-1} u\left(t_{2}\right)-D_{q}^{\alpha-1} u\left(t_{1}\right)<\varepsilon\right|, \quad i=2, \ldots, n-1 .
\end{aligned}
$$

Proof. The method is similar to the proof of Lemma 2.3 in [26], so we omit the details.

Lemma 9. If condition $\left(\mathrm{H}_{2}\right)$ holds, then for $u \in X$, we have

$$
\int_{0}^{+\infty}\left|f\left(t, u(t), D_{q}^{\alpha-1} u(t), \ldots, D_{q}^{\alpha-(n-1)} u(t)\right)\right| \mathrm{d}_{q} t \leq a_{0}+\sum_{i=1}^{n} a_{i}\|u\|^{\lambda_{i}} .
$$

Proof. For $u \in X$, by condition $\left(H_{2}\right)$, we have

$$
\begin{aligned}
& \int_{0}^{+\infty}\left|f\left(t, u(t), D_{q}^{\alpha-1} u(t), \ldots, D_{q}^{\alpha-(n-1)} u(t)\right)\right| \mathrm{d}_{q} t \leq \int_{0}^{+\infty} a_{0}(t) \mathrm{d}_{q} t+\int_{0}^{+\infty} a_{1}(t)|u|^{\lambda_{1}} \mathrm{~d}_{q} t+\int_{0}^{+\infty} \sum_{i=1}^{n} a_{i}(t)\left|D_{q}^{\alpha-i} u\right|^{\lambda_{i}} \mathrm{~d}_{q} t \\
& \quad=a_{0}+\int_{0}^{+\infty}\left(1+t^{\alpha-1}\right)^{\lambda_{1}} a_{1}(t) \frac{|u|^{\lambda_{1}}}{\left(1+t^{\alpha-1}\right)^{\lambda_{1}}} \mathrm{~d}_{q} t+\int_{0}^{+\infty} a_{2}(t)\left|D_{q}^{\alpha-1} u\right|^{\lambda_{2}} \mathrm{~d}_{q} t+\int_{0}^{+\infty} \sum_{i=2}^{n-1} a_{i+1}(t)\left(1+t^{i-1}\right)^{\lambda_{i+1}} \frac{\left|D_{q}^{\alpha-i} u(t)\right|^{\lambda_{i+1}}}{\left(1+t^{i-1}\right)^{\lambda_{i+1}}} \mathrm{~d}_{q} t \\
& \leq a_{0}+a_{1}\|u\|^{\lambda_{1}}+\cdots+a_{n}\|u\|^{\lambda_{n}}=a_{0}+\sum_{i=1}^{n} a_{i}\|u\|^{\lambda_{i}} .
\end{aligned}
$$

Lemma 10. If condition $\left(\mathrm{H}_{3}\right)$ holds, then for $u \in X$, we have

$$
\int_{0}^{+\infty}\left|f\left(t, u(t), D_{q}^{\alpha-1} u(t), \ldots, D_{q}^{\alpha-(n-1)} u(t)\right)\right| \mathrm{d}_{q} t \leq \kappa+\sum_{i=1}^{n} b_{i}\|u\| .
$$

Proof. For $u \in X$, by condition $\left(H_{3}\right)$, we have

$$
\begin{aligned}
& \int_{0}^{+\infty}\left|f\left(t, u(t), D_{q}^{\alpha-1} u(t), \ldots, D_{q}^{\alpha-(n-1)} u(t)\right)\right| d_{q} t \leq \int_{0}^{+\infty}\left|f\left(t, u(t), D_{q}^{\alpha-1} u(t), \ldots, D_{q}^{\alpha-(n-1)}-f(t, 0, \ldots, 0)\right)\right| \mathrm{d}_{q} t \\
& \quad+\int_{0}^{+\infty}|f(t, 0, \ldots, 0)| \mathrm{d}_{q} t \leq \int_{0}^{+\infty} b_{1}(t)|u(t)| \mathrm{d}_{q} t+\int_{0}^{+\infty} b_{2}(t)\left|D_{q}^{\alpha-1} u(t) \mathrm{d}_{q} t+\cdots+\int_{0}^{+\infty} b_{n}(t)\right| D_{q}^{\alpha-(n-1)} u(t) \mathrm{d}_{q} t \\
& =\int_{0}^{+\infty}\left(1+t^{\alpha-1}\right) \frac{|u(t)|}{1+t^{\alpha-1}} b_{1}(t) \mathrm{d}_{q} t+\int_{0}^{+\infty} b_{2}(t)\left|D_{q}^{\alpha-1} u(t)\right| \mathrm{d}_{q} t+\int_{0}^{+\infty} \sum_{i=2}^{n-1} b_{i+1} \frac{\left|D_{q}^{\alpha-i} u(t)\right|}{1+t^{i-1}} \mathrm{~d}_{q} t+\int_{0}^{+\infty}|f(t, 0, \ldots, 0)| \mathrm{d}_{q} t \leq \sum_{i=1}^{n} b_{i}\|u\|+\kappa
\end{aligned}
$$


Define the cone $P \subset X$ by

$$
P=\left\{\begin{array}{c}
u \in X \mid u(t) \geq 0 \\
D_{q}^{\alpha-i} u(t) \geq 0, \quad t \in J, i=1,2, \ldots, n-1
\end{array}\right\}
$$

and define the operator $\mathscr{T}: P \mapsto P$ as follows:

$$
\mathscr{T} u(t)=\int_{0}^{+\infty} G(t, q s) f\left(s, u(s), D_{q}^{\alpha-1} u(s), \ldots, D_{q}^{\alpha-(n-1)} u(s)\right) \mathrm{d}_{q} s .
$$

We also define

$$
D_{q}^{\alpha-i} \mathscr{T} u(t)=\int_{0}^{+\infty} G_{i}^{*}(t, q s) f\left(s, u(s), D_{q}^{\alpha-1} u(s), \ldots, D_{q}^{\alpha-(n-1)} u(s)\right) \mathrm{d}_{q} s .
$$

It is easy to see problem (1) has a solution, if and only if the operator $\mathscr{T}$ has a fixed point.

Lemma 11. If conditions $\left(H_{1}\right)$ and $\left(H_{2}\right)$ hold, then the operator $\mathscr{T}: P \mapsto P$ is completely continuous.

Proof. It is easy to see $\mathscr{T}: P \mapsto P$. Since $G(t, q s) \geq 0$ and $f \geq 0$, we know $\mathscr{T}(u)(t) \geq 0$, for $u \in P, t \in J$. In the following, we divide the proof into four steps:

Step 1: we show that $\mathscr{T}$ is uniformly bounded. Let $\Omega$ be any bounded subset of $P$, i.e., there exists $M>0$ such that $\|u\| \leq M$ for each $u \in \Omega$. We only need to show that $\mathscr{T} u$ is bounded in $P$. For $u \in \Omega$, by Lemmas 5 and 8 , we have

$$
\begin{aligned}
\left\|\frac{\mathscr{T} u}{1+t^{\alpha-1}}\right\|_{\infty} & =\sup _{t \in J}\left|\frac{\mathscr{T} u}{1+t^{\alpha-1}}\right|=\sup _{t \in J}\left|\int_{0}^{+\infty} \frac{G(t, q s)}{1+t^{\alpha-1}} f\left(s, u(s), D_{q}^{\alpha-1} u(s), \ldots, D_{q}^{\alpha-(n-1)} u(s)\right) \mathrm{d}_{q} s\right| \\
& \leq \frac{1}{\Gamma_{q}(\alpha)-\Lambda}\left(a_{0}+\sum_{k=1}^{n} a_{k}\|u\|^{\lambda_{k}}\right) \leq \frac{1}{\Gamma_{q}(\alpha)-\Lambda}\left(a_{0}+\sum_{k=1}^{n} a_{k} M^{\lambda_{k}}\right) .
\end{aligned}
$$

By Lemmas 5, 7, and 9, we have

$$
\begin{aligned}
\left\|\frac{D_{q}^{\alpha-i} \mathscr{T} u}{1+t^{i-1}}\right\|_{\infty} & =\sup _{t \in J}\left|\frac{D_{q}^{\alpha-i} \mathscr{T} u}{1+t^{i-1}}\right|=\sup _{t \in J}\left|\int_{0}^{+\infty} \frac{G_{i}^{*}(t, q s)}{1+t^{i-1}} f\left(s, u(s), D_{q}^{\alpha-1} u(s), \ldots, D_{q}^{\alpha-(n-1)} u(s)\right) \mathrm{d}_{q} s\right| \\
& \leq \frac{\Gamma_{q}(\alpha)}{\Gamma_{q}(i)\left(\Gamma_{q}(\alpha)-\Lambda\right)}\left(a_{0}+\sum_{k=1}^{n} a_{k}\|u\|^{\lambda_{k}}\right), \quad i=2,3, \ldots, n-1 \\
& \leq \frac{\Gamma_{q}(\alpha)}{\Gamma_{q}(i)\left(\Gamma_{q}(\alpha)-\Lambda\right)}\left(a_{0}+\sum_{k=1}^{n} a_{k} M^{\lambda_{k}}\right), \quad i=2,3, \ldots, n-1, \\
\left\|D_{q}^{\alpha-1} \mathscr{T} u\right\|_{\infty} & =\sup _{t \in J}\left|\int_{0}^{+\infty} G_{1}^{*}(t, q s) f\left(s, u(s), D_{q}^{\alpha-1} u(s), \ldots, D_{q}^{\alpha-(n-1)} u(s)\right) \mathrm{d}_{q} s\right| \\
& \leq \frac{\Gamma_{q}(\alpha)}{\Gamma_{q}(\alpha)-\Lambda}\left(a_{0}+\sum_{k=1}^{n} a_{k}\|u\|^{\lambda_{k}}\right) \leq \frac{\Gamma_{q}(\alpha)}{\Gamma_{q}(\alpha)-\Lambda}\left(a_{0}+\sum_{k=1}^{n} a_{k} M^{\lambda_{k}}\right) .
\end{aligned}
$$

From (54)-(56), we obtain

$$
\|\mathscr{T} u\| \leq\left(a_{0}+\sum_{k=1}^{n} a_{k} M^{\lambda_{k}}\right) \max \left\{\frac{1}{\Gamma_{q}(\alpha)-\Lambda}, \frac{\Gamma_{q}(\alpha)}{\Gamma_{q}(\alpha)-\Lambda}, \frac{\Gamma_{q}(\alpha)}{\Gamma_{q}(i)\left(\Gamma_{q}(\alpha)-\Lambda\right)}, \quad i=2,3, \ldots, n-1\right\}
$$


which means that $\mathscr{T} u$ is uniformly bounded.

Step 2: we show that the operator $\mathscr{T}$ is equicontinuous for all $u \in \Omega$ on any compact interval $I$ of $J$. For any given $T>0, t_{1}, t_{2} \in[0, T]$ and $t_{2}>t_{1}, u \in \Omega$, we have

$$
\begin{aligned}
\left|\frac{\mathscr{T} u\left(t_{2}\right)}{1+t_{2}^{\alpha-1}}-\frac{\mathscr{T} u\left(t_{1}\right)}{1+t_{1}^{\alpha-1}}\right|= & \left|\int_{0}^{+\infty}\left(\frac{G\left(t_{2}, q s\right)}{1+t_{2}^{\alpha-1}}-\frac{G\left(t_{1}, q s\right)}{1+t_{1}^{\alpha-1}}\right) f\left(s, u(s), D_{q}^{\alpha-1} u(s), \ldots, D_{q}^{\alpha-(n-1)} u(s)\right) \mathrm{d}_{q} s\right| \\
\leq & \int_{0}^{+\infty}\left|\frac{G_{1}\left(t_{2}, q s\right)}{1+t_{2}^{\alpha-1}}-\frac{G_{1}\left(t_{1}, q s\right)}{1+t_{2}^{\alpha-1}}\right|\left|f\left(s, u(s), D_{q}^{\alpha-1} u(s), \ldots, D_{q}^{\alpha-(n-1)} u(s)\right)\right| \mathrm{d}_{q} s \\
& +\int_{0}^{+\infty}\left|\frac{G_{1}\left(t_{2}, q s\right)}{1+t_{2}^{\alpha-1}}-\frac{G_{1}\left(t_{1}, q s\right)}{1+t_{1}^{\alpha-1}}\right|\left|f\left(s, u(s), D_{q}^{\alpha-1} u(s), \ldots, D_{q}^{\alpha-(n-1)} u(s)\right)\right| \mathrm{d}_{q} s \\
& +\int_{0}^{+\infty}\left|\frac{G_{2}\left(t_{2}, q s\right)}{1+t_{2}^{\alpha-1}}-\frac{G_{2}\left(t_{1}, q s\right)}{1+t_{1}^{\alpha-1}}\right|\left|f\left(s, u(s), D_{q}^{\alpha-1} u(s), \ldots, D_{q}^{\alpha-(n-1)} u(s)\right)\right| \mathrm{d}_{q} s .
\end{aligned}
$$

By Lemmas 5 and 6, we have

$$
\begin{aligned}
& \int_{0}^{+\infty}\left|\frac{G_{2}\left(t_{2}, q s\right)}{1+t_{2}^{\alpha-1}}-\frac{G_{2}\left(t_{1}, q s\right)}{1+t_{1}^{\alpha-1}}\right|\left|f\left(s, u(s), D_{q}^{\alpha-1} u(s), \ldots, D_{q}^{\alpha-(n-1)} u(s)\right)\right| \mathrm{d}_{q} s \\
& \quad \leq \int_{0}^{+\infty} \frac{1}{\left(\Gamma_{q}(\alpha)-\Lambda\right)^{2}}\left|\frac{t_{2}^{\alpha-1}-t_{1}^{\alpha-1}}{\left(1+t_{1}^{\alpha-1}\right)\left(1+t_{2}^{\alpha-1}\right)}\right|\left|f\left(s, u(s), D_{q}^{\alpha-1} u(s), \ldots, D_{q}^{\alpha-(n-1)} u(s)\right)\right| \mathrm{d}_{q} s \longrightarrow 0 .
\end{aligned}
$$

On the other hand, for all $u \in \Omega, t_{1} \longrightarrow t_{2}$, we have

$$
\begin{aligned}
& \int_{0}^{+\infty}\left|\frac{G_{1}\left(t_{2}, q s\right)}{1+t_{2}^{\alpha-1}}-\frac{G_{1}\left(t_{1}, q s\right)}{1+t_{2}^{\alpha-1}}\right|\left|f\left(s, u(s), D_{q}^{\alpha-1} u(s), \ldots, D_{q}^{\alpha-(n-1)} u(s)\right)\right| \mathrm{d}_{q} s \\
& \quad \leq \int_{0}^{t_{1}}\left|\frac{t_{2}^{\alpha-1}-t_{1}^{\alpha-1}-\left(t_{2}-q s\right)^{(\alpha-1)}+\left(t_{1}-q s\right)^{(\alpha-1)}}{\Gamma_{q}(\alpha)\left(1+t_{2}^{\alpha-1}\right)}\right|\left|f\left(s, u(s), D_{q}^{\alpha-1} u(s), \ldots, D_{q}^{\alpha-(n-1)} u(s)\right)\right| \mathrm{d}_{q} s \\
& +\int_{t_{1}}^{t_{2}}\left|\frac{t_{2}^{\alpha-1}-t_{1}^{\alpha-1}-\left(t_{2}-q s\right)^{(\alpha-1)}}{\Gamma_{q}(\alpha)\left(1+t_{2}^{\alpha-1}\right)}\right|\left|f\left(s, u(s), D_{q}^{\alpha-1} u(s), \ldots, D_{q}^{\alpha-(n-1)} u(s)\right)\right| \mathrm{d}_{q} s \\
& \quad+\int_{t_{2}}^{+\infty}\left|\frac{t_{2}^{\alpha-1}-t_{1}^{\alpha-1}}{\Gamma_{q}(\alpha)\left(1+t_{2}^{\alpha-1}\right)}\right|\left|f\left(s, u(s), D_{q}^{\alpha-1} u(s), \ldots, D_{q}^{\alpha-(n-1)} u(s)\right)\right| \mathrm{d}_{q} s \longrightarrow 0 .
\end{aligned}
$$


Similar to (60), for all $u \in \Omega, t_{1} \longrightarrow t_{2}$, we have

$$
\begin{aligned}
& \int_{0}^{+\infty}\left|\frac{G_{1}\left(t_{1}, q s\right)}{1+t_{2}^{\alpha-1}}-\frac{G_{1}\left(t_{1}, q s\right)}{1+t_{1}^{\alpha-1}}\right|\left|f\left(s, u(s), D_{q}^{\alpha-1} u(s), \ldots, D_{q}^{\alpha-(n-1)} u(s)\right)\right| \mathrm{d}_{q} s \\
& \quad \leq \int_{0}^{t_{1}} \frac{t_{1}^{\alpha-1}-\left(t_{1}-q s\right)^{(\alpha-1)}}{\Gamma_{q}(\alpha)} \frac{t_{2}^{\alpha-1}-t_{1}^{\alpha-1}}{\left(1+t_{1}^{\alpha-1}\right)\left(1+t_{2}^{\alpha-1}\right)} \| \begin{array}{c}
f\left(s, u(s), D_{q}^{\alpha-1} u(s), \ldots, D_{q}^{\alpha-(n-1)} u(s)\right) \mathrm{d}_{q} s \| \\
\quad+\int_{t_{1}}^{+\infty} \frac{t_{1}^{\alpha-1}}{\Gamma_{q}(\alpha)} \frac{t_{2}^{\alpha-1}-t_{1}^{\alpha-1}}{\left(1+t_{1}^{\alpha-1}\right)\left(1+t_{2}^{\alpha-1}\right)} \|
\end{array} \\
& \left|f\left(s, u(s), D_{q}^{\alpha-1} u(s), \ldots, D_{q}^{\alpha-(n-1)} u(s)\right)\right| \mathrm{d}_{q} s \longrightarrow 0 .
\end{aligned}
$$

Together (59)-(61), which implies that Note that, from (53) and Lemma 5, we have $\left(\mathscr{T} u(t) / 1+t^{\alpha-1}\right)$ is equicontinuous on any compact intervals of $[0, \infty)$.

$$
\begin{aligned}
D_{q}^{\alpha-1} \mathscr{T} u(t) & =\int_{0}^{+\infty} G_{1}^{*}(t, q s) f\left(s, u(s), D_{q}^{\alpha-1} u(s), \ldots, D_{q}^{\alpha-(n-1)} u(s)\right) \mathrm{d}_{q} s \\
G_{1}^{*}(t, q s) & =G_{11}^{*}(t, q s)+G_{12}^{*}(t, q s)
\end{aligned}
$$

where

$$
\begin{aligned}
& G_{11}^{*}(t, q s)= \begin{cases}0, & 0 \leq s \leq t \leq+\infty \\
1, & 0 \leq t \leq s \leq+\infty\end{cases} \\
& G_{12}^{*}(t, q s)=\frac{\Gamma_{q}(\alpha)}{\Gamma_{q}(\alpha)-\Lambda} \int_{0}^{+\infty} g(t) G_{1}(t, q s) \mathrm{d}_{q} t .
\end{aligned}
$$

which means $G_{1}^{*}(t, q s)$ does not rely on $t$. Hence, $D_{q}^{\alpha-1} \mathscr{T} u$ is equicontinuous on any compact intervals of $J$.

In the following, we show that $D_{q}^{\alpha-i} \mathscr{T} u$, $i=2,3, \ldots, n-1$, is equicontinuous on any compact intervals of $J$. In fact, for any given $T>0, t_{1}, t_{2} \in[0, T]$ and $t_{2}>t_{1}, u \in \Omega$, we have

$$
\begin{aligned}
\left|\frac{D_{q}^{\alpha-i} \mathscr{T} u\left(t_{2}\right)}{1+t_{2}^{i-1}}-\frac{D_{q}^{\alpha-i} \mathscr{T} u\left(t_{1}\right)}{1+t_{1}^{i-1}}\right|= & \left|\int_{0}^{+\infty}\left(\frac{G_{i}^{*}\left(t_{2}, q s\right)}{1+t_{2}^{i-1}}-\frac{G_{i}^{*}\left(t_{1}, q s\right)}{1+t_{1}^{i-1}}\right) f\left(s, u(s), D_{q}^{\alpha-1} u(s), \ldots, D_{q}^{\alpha-(n-1)} u(s)\right) \mathrm{d}_{q} s\right| \\
\leq & \int_{0}^{+\infty}\left|\frac{G_{i 1}^{*}\left(t_{2}, q s\right)}{1+t_{2}^{i-1}}-\frac{G_{i 1}^{*}\left(t_{1}, q s\right)}{1+t_{2}^{i-1}}\right|\left|f\left(s, u(s), D_{q}^{\alpha-1} u(s), \ldots, D_{q}^{\alpha-(n-1)} u(s)\right)\right| \mathrm{d}_{q} s \\
& +\int_{0}^{+\infty}\left|\frac{G_{i 1}^{*}\left(t_{1}, q s\right)}{1+t_{2}^{i-1}}-\frac{G_{i 1}^{*}\left(t_{1}, q s\right)}{1+t_{1}^{i-1}}\right|\left|f\left(s, u(s), D_{q}^{\alpha-1} u(s), \ldots, D_{q}^{\alpha-(n-1)} u(s)\right)\right| \mathrm{d}_{q} s \\
& +\int_{0}^{+\infty}\left|\frac{G_{i 2}^{*}\left(t_{2}, q s\right)}{1+t_{2}^{i-1}}-\frac{G_{i 2}^{*}\left(t_{1}, q s\right)}{1+t_{1}^{i-1}}\right|\left|f\left(s, u(s), D_{q}^{\alpha-1} u(s), \ldots, D_{q}^{\alpha-(n-1)} u(s)\right)\right| \mathrm{d}_{q} s .
\end{aligned}
$$

Similar to (59)-(61), we obtain

$$
\left|\frac{D_{q}^{\alpha-i} \mathscr{T} u\left(t_{2}\right)}{1+t_{2}^{i-1}}-\frac{D_{q}^{\alpha-i} \mathscr{T} u\left(t_{1}\right)}{1+t_{1}^{i-1}}\right| \longrightarrow 0 \text {, as } t_{1} \longrightarrow t_{2}
$$

Inequalities (59)-(64) imply that the operator $\mathscr{T}$ is equicontinuous on any compact intervals of $J$.

Step 3: we show that $\mathscr{T}$ is equiconvergent at $\infty$. We need to prove that, for $\forall \varepsilon>0, \exists T(\varepsilon)>0$, such that, for any $t_{1}, t_{2}>T(\varepsilon)$, the following inequalities hold: 
Journal of Mathematics

11

$$
\begin{aligned}
& \left|\frac{\mathscr{T} u\left(t_{2}\right)}{1+t_{2}^{\alpha-1}}-\frac{\mathscr{T} u\left(t_{1}\right)}{1+t_{1}^{\alpha-1}}\right|<\varepsilon, \\
& \left|D_{q}^{\alpha-1} \mathscr{T} u\left(t_{2}\right)-D_{q}^{\alpha-1} \mathscr{T} u\left(t_{1}\right)\right|<\varepsilon, \\
& \left|\frac{D_{q}^{\alpha-i} \mathscr{T} u\left(t_{2}\right)}{1+t_{2}^{i-1}}-\frac{D_{q}^{\alpha-i} \mathscr{T} u\left(t_{1}\right)}{1+t_{1}^{i-1}}\right|<\varepsilon, \quad i=2,3, \ldots, n-1 .
\end{aligned}
$$

Note that, since the function $G_{1}^{*}(t, s)$ does not rely on $t$, it is easy to infer that

$$
\left|D_{q}^{\alpha-1} \mathscr{T} u\left(t_{2}\right)-D_{q}^{\alpha-1} \mathscr{T} u\left(t_{1}\right)\right|<\varepsilon,
$$

ie., $D_{q}^{\alpha-1} \mathscr{T} u(t)$ is equiconvergent at $+\infty$.
By $\left(H_{1}\right)$ and Lemma 9, for any $\varepsilon>0$, there exists $a>0$ such that

$$
\int_{a}^{+\infty} f\left(s, u(s), D_{q}^{\alpha-1} u(s), \ldots, D_{q}^{\alpha-(n-1)} u(s)\right) \mathrm{d}_{q} s<\varepsilon .
$$

On the other hand, since

$$
\begin{aligned}
\lim _{t \longrightarrow+\infty} \frac{t^{\gamma}}{1+t^{\gamma}} & =1, \\
\gamma & \in\{\alpha-1, i-1\}, \quad i=2, \ldots, n-1 .
\end{aligned}
$$

There exists sufficiently large $T_{1}>0$ such that for any $t_{1}, t_{2}>T_{1}$, we have

$$
\left|\frac{t_{2}^{\alpha-1}}{1+t_{2}^{\alpha-1}}-\frac{t_{1}^{\alpha-1}}{1+t_{1}^{\alpha-1}}\right|<\varepsilon \text { and }\left|\frac{t_{2}^{i-1}}{1+t_{2}^{i-1}}-\frac{t_{1}^{i-1}}{1+t_{1}^{i-1}}\right|<\varepsilon, \quad i=2, \ldots, n-1
$$

Also because $\lim _{t \rightarrow+\infty}\left((t-a)^{(\theta-1)} / 1+t^{\theta-1}\right)=1$, $\theta \in\{\alpha, i\}, i=2, \ldots, n-1$, there exists sufficiently large
$T_{2}>a$ such that, for any $t_{1}, t_{2}>T_{3}$ and $0 \leq q s<s \leq a$, we have

$$
\left|\frac{\left(t_{2}-q s\right)^{(\alpha-1)}}{1+t_{2}^{\alpha-1}}-\frac{\left(t_{1}-q s\right)^{(\alpha-1)}}{1+t_{1}^{\alpha-1}}\right|<\varepsilon \text { and }\left|\frac{\left(t_{2}-q s\right)^{(i-1)}}{1+t_{2}^{i-1}}-\frac{\left(t_{1}-q s\right)^{(i-1)}}{1+t_{1}^{i-1}}\right|<\varepsilon, \quad i=2, \ldots, n-1 .
$$

Choose $T>\max \left\{T_{1}, T_{2}\right\}$, for any $t_{1}, t_{2}>T$, and let $F(s):=f\left(s, u(s), D_{q}^{\alpha-1} u(s), \ldots, D_{q}^{\alpha-(n-1)} u(s)\right), \quad$ and $t_{1} \longrightarrow t_{2}$. For convenience, we denote we obtain

$$
\begin{aligned}
& \left|\frac{\mathscr{T} u\left(t_{2}\right)}{1+t_{2}^{\alpha-1}}-\frac{\mathscr{T} u\left(t_{1}\right)}{1+t_{1}^{\alpha-1}}\right|=\left|\int_{0}^{+\infty}\left(\frac{G\left(t_{2}, q s\right)}{1+t_{2}^{\alpha-1}}-\frac{G\left(t_{1}, q s\right)}{1+t_{1}^{\alpha-1}}\right) F(s) \mathrm{d}_{q} s\right| \\
& \leq\left|\int_{0}^{+\infty}\left(\frac{G_{1}\left(t_{2}, q s\right)}{1+t_{2}^{\alpha-1}}-\frac{G_{1}\left(t_{1}, q s\right)}{1+t_{2}^{\alpha-1}}\right) F(s) \mathrm{d}_{q} s\right|+\left|\int_{0}^{+\infty}\left(\frac{G_{1}\left(t_{2}, q s\right)}{1+t_{2}^{\alpha-1}}-\frac{G_{1}\left(t_{1}, q s\right)}{1+t_{1}^{\alpha-1}}\right) F(s) \mathrm{d}_{q} s\right| \\
& \quad+\left|\int_{0}^{+\infty}\left(\frac{G_{2}\left(t_{2}, q s\right)}{1+t_{2}^{\alpha-1}}-\frac{G_{2}\left(t_{1}, q s\right)}{1+t_{2}^{\alpha-1}}\right) F(s) \mathrm{d}_{q} s\right| \\
& \leq \frac{1}{\Gamma_{q}(\alpha)} \int_{0}^{t_{1}}\left(\left|\frac{t_{2}^{\alpha-1}}{1+t_{2}^{\alpha-1}}-\frac{t_{1}^{\alpha-1}}{1+t_{1}^{\alpha-1}}\right|+\left|\frac{\left(t_{2}-q s\right)^{\alpha-1}}{1+t_{2}^{\alpha-1}}-\frac{\left(t_{1}-q s\right)^{\alpha-1}}{1+t_{1}^{\alpha-1}}\right|\right) F(s) \mathrm{d}_{q} s \\
& \quad+\frac{1}{\Gamma_{q}(\alpha)} \int_{t_{1}}^{t_{2}}\left(\left|\frac{t_{2}^{\alpha-1}}{1+t_{2}^{\alpha-1}}-\frac{t_{1}^{\alpha-1}}{1+t_{1}^{\alpha-1}}\right|+\left|\frac{\left(t_{2}-q s\right)^{\alpha-1}}{1+t_{2}^{\alpha-1}}\right|\right) F(s) \mathrm{d}_{q} s+\frac{1}{\Gamma_{q}(\alpha)} \int_{t_{2}}^{+\infty}\left|\frac{t_{2}^{\alpha-1}}{1+t_{2}^{\alpha-1}}-\frac{t_{1}^{\alpha-1}}{1+t_{1}^{\alpha-1} \mid}\right| F(s) \mathrm{d}_{q} s \\
& \quad+\frac{1}{\left(\Gamma_{q}(\alpha)-\Lambda\right)^{2}}\left|\frac{t_{2}^{\alpha-1}}{1+t_{2}^{\alpha-1}}-\frac{t_{1}^{\alpha-1}}{1+t_{1}^{\alpha-1}}\right| \int_{0}^{+\infty} F(s) \mathrm{d}_{q} s .
\end{aligned}
$$


By (68) and (72), we obtain

$$
\begin{aligned}
& \left|\frac{\mathscr{T} u\left(t_{2}\right)}{1+t_{2}^{\alpha-1}}-\frac{\mathscr{T} u\left(t_{1}\right)}{1+t_{1}^{\alpha-1}}\right| \leq \frac{1}{\Gamma_{q}(\alpha)} \int_{0}^{a}\left(\left|\frac{t_{2}^{\alpha-1}}{1+t_{2}^{\alpha-1}}-\frac{t_{1}^{\alpha-1}}{1+t_{1}^{\alpha-1}}\right|+\left|\frac{\left(t_{2}-q s\right)^{\alpha-1}}{1+t_{2}^{\alpha-1}}-\frac{\left(t_{1}-q s\right)^{\alpha-1}}{1+t_{1}^{\alpha-1}}\right|\right) F(s) \mathrm{d}_{q} s \\
& +\frac{1}{\Gamma_{q}(\alpha)} \int_{a}^{+\infty}\left(\left|\frac{t_{2}^{\alpha-1}}{1+t_{2}^{\alpha-1}}-\frac{t_{1}^{\alpha-1}}{1+t_{1}^{\alpha-1}}\right|+\left|\frac{\left(t_{2}-q s\right)^{\alpha-1}}{1+t_{2}^{\alpha-1}}-\frac{\left(t_{1}-q s\right)^{\alpha-1}}{1+t_{1}^{\alpha-1}}\right|\right) F(s) \mathrm{d}_{q} s \\
& +\frac{1}{\Gamma_{q}(\alpha)} \int_{a}^{+\infty}\left(\left|\frac{t_{2}^{\alpha-1}}{1+t_{2}^{\alpha-1}}-\frac{t_{1}^{\alpha-1}}{1+t_{1}^{\alpha-1}}\right|+\left|\frac{\left(t_{2}-q s\right)^{\alpha-1}}{1+t_{2}^{\alpha-1}}\right|\right) F(s) \mathrm{d}_{q} s \\
& +\frac{1}{\Gamma_{q}(\alpha)} \int_{a}^{+\infty}\left|\frac{t_{2}^{\alpha-1}}{1+t_{2}^{\alpha-1}}-\frac{t_{1}^{\alpha-1}}{1+t_{1}^{\alpha-1}}\right| F(s) \mathrm{d}_{q} s+\frac{1}{\left(\Gamma_{q}(\alpha)-\Lambda\right)^{2}}\left|\frac{t_{2}^{\alpha-1}}{1+t_{2}^{\alpha-1}}-\frac{t_{1}^{\alpha-1}}{1+t_{1}^{\alpha-1}}\right| \int_{0}^{+\infty} F(s) \mathrm{d}_{q} s \\
& \leq \frac{1}{\Gamma_{q}(\alpha)} \max _{s \in[0, a], u \in \Omega} F(s) \int_{0}^{a}\left(\left|\frac{t_{2}^{\alpha-1}}{1+t_{2}^{\alpha-1}}-\frac{t_{1}^{\alpha-1}}{1+t_{1}^{\alpha-1}}\right|+\left|\frac{\left(t_{2}-q s\right)^{\alpha-1}}{1+t_{2}^{\alpha-1}}-\frac{\left(t_{1}-q s\right)^{\alpha-1}}{1+t_{1}^{\alpha-1}}\right|\right) \mathrm{d}_{q} s \\
& +\frac{9}{\Gamma_{q}(\alpha)} \int_{a}^{+\infty} F(s) \mathrm{d}_{q} s+\frac{\varepsilon}{\left(\Gamma_{q}(\alpha)-\Lambda\right)^{2}} \int_{0}^{+\infty} F(s) \mathrm{d}_{q} s \\
& \leq \frac{2 a \varepsilon}{\Gamma_{q}(\alpha)} \max _{s \in[0, a], u \in \Omega} F(s)+\frac{9 \varepsilon}{\Gamma_{q}(\alpha)}+\frac{\varepsilon}{\left(\Gamma_{q}(\alpha)-\Lambda\right)^{2}} \int_{0}^{+\infty} F(s) \mathrm{d}_{q} s \\
& \leq\left(\frac{2 a}{\Gamma_{q}(\alpha)} \max _{s \in[0, a], u \in \Omega} F(s)+\frac{9}{\Gamma_{q}(\alpha)}+\frac{a_{0}+\sum_{k=1}^{n} a_{k} M^{\lambda_{k}}}{\left(\Gamma_{q}(\alpha)-\Lambda\right)^{2}}\right) \mathcal{\varepsilon} .
\end{aligned}
$$

Similar to (72) and (73), we obtain

$$
\left|\frac{D_{q}^{\alpha-i} \mathscr{T} u\left(t_{2}\right)}{1+t_{2}^{i-1}}-\frac{D_{q}^{\alpha-i} \mathscr{T} u\left(t_{1}\right)}{1+t_{1}^{i-1}}\right| \leq\left(\frac{2 a}{\Gamma_{q}(i)} \max _{s \in[0, a], u \in \Omega} F(s)+\frac{9}{\Gamma_{q}(i)}+\frac{\left(a_{0}+\sum_{k=1}^{n} a_{k} M^{\lambda_{k}}\right) \Gamma_{q}(\alpha) \Lambda}{\left(\Gamma_{q}(i) \Gamma_{q}(\alpha)-\Lambda\right)^{2}}\right) \varepsilon
$$

From (67), (73), and (74), we obtain $\mathscr{T}$ is equiconvergent at $\infty$.

The above three steps and Lemma 8 imply that the operator $\mathscr{T}: P \longrightarrow P$ is relatively compact.

Step 4: we show that the operator $\mathscr{T}: P \longrightarrow P$ is continuous. For $u_{m} \in P$, such that $u_{m} \longrightarrow u$ as $m \longrightarrow \infty$, and we need to show that $\left\|\mathscr{T} u_{m}-\mathscr{T} u\right\| \longrightarrow 0$ as $m \longrightarrow \infty$.

By Lemma 6 , the continuity of function $f$, and the Lebesgue dominated convergence theorem, we obtain

$$
\begin{aligned}
\left\|\frac{\mathscr{T} u_{m}}{1+t^{\alpha-1}}-\frac{\mathscr{T} u}{1+t^{\alpha-1}}\right\|_{\infty} & =\sup _{t \in J}\left|\int_{0}^{+\infty} \frac{G(t, q s)}{1+t^{\alpha-1}}\left(\begin{array}{c}
\left(f\left(s, u_{m}(s), D_{q}^{\alpha-1} u_{m}(s), \ldots, D_{q}^{\alpha-(n-1)} u_{m}(s)\right)\right. \\
-f\left(s, u(s), D_{q}^{\alpha-1} u(s), \ldots, D_{q}^{\alpha-(n-1)} u(s)\right)
\end{array}\right) \mathrm{d}_{q} s\right| \\
& \leq \frac{1}{\Gamma_{q}(\alpha)-\Lambda} \int_{0}^{+\infty}\left|\begin{array}{c}
f\left(s, u_{m}(s), D_{q}^{\alpha-1} u_{m}(s), \ldots, D_{q}^{\alpha-(n-1)} u_{m}(s)\right) \\
-f\left(s, u(s), D_{q}^{\alpha-1} u(s), \ldots, D_{q}^{\alpha-(n-1)} u(s)\right)
\end{array}\right| \mathrm{d}_{q} s \longrightarrow 0, \text { as } m \longrightarrow \infty .
\end{aligned}
$$


Similar to (75), by Lemmas 7 and 9, we get

$$
\begin{aligned}
& \left\|D_{q}^{\alpha-1} \mathscr{T} u_{m}-D_{q}^{\alpha-1} \mathscr{T} u\right\|_{\infty} \leq \frac{\Gamma_{q}(\alpha)}{\Gamma_{q}(\alpha)-\Lambda} \int_{0}^{+\infty}\left|\begin{array}{c}
f\left(s, u_{m}(s), D_{q}^{\alpha-1} u_{m}(s), \ldots, D_{q}^{\alpha-(n-1)} u_{m}(s)\right) \\
-f\left(s, u(s), D_{q}^{\alpha-1} u(s), \ldots, D_{q}^{\alpha-(n-1)} u(s)\right)
\end{array}\right| \mathrm{d}_{q} s \longrightarrow 0, \text { as } m \longrightarrow \infty \\
& \left\|\frac{D_{q}^{\alpha-i} \mathscr{T} u_{m}}{1+t^{i-1}}-\frac{D_{q}^{\alpha-i} \mathscr{T} u}{1+t^{i-1}}\right\|_{\infty} \leq \frac{\Gamma_{q}(\alpha)}{\Gamma_{q}(i)\left(\Gamma_{q}(\alpha)-\Lambda\right)} \\
& \int_{0}^{+\infty}\left|f\left(s, u_{m}(s), D_{q}^{\alpha-1} u_{m}(s), \ldots, D_{q}^{\alpha-(n-1)} u_{m}(s)\right)-f\left(s, u(s), D_{q}^{\alpha-1} u(s), \ldots, D_{q}^{\alpha-(n-1)} u(s)\right)\right| \mathrm{d}_{q} s \longrightarrow 0, \text { as } m \longrightarrow \infty
\end{aligned}
$$

for all $i=2,3, \ldots, n-1$.

From (75)-(77), we have

$$
\left\|\mathscr{T} u_{m}-\mathscr{T} u\right\| \longrightarrow 0, \text { as } m \longrightarrow \infty
$$

which means that the operator $\mathscr{T}$ is continuous.

In view of all the above arguments, we deduce that the operator $\mathscr{T}: P \longrightarrow P$ is completely continuous. This completes the proof.

\section{Main Results}

In this section, we give the main results of this paper. For convenience, we denote

$$
\rho_{i}=\frac{1}{\Gamma_{q}(i)\left(\Gamma_{q}(\alpha)-\Lambda\right)}, \quad i=1,2, \ldots, n-1 .
$$

Let

$$
L=\max \left\{\rho_{1}, \rho_{1} \Gamma_{q}(\alpha), \rho_{2} \Gamma_{q}(\alpha), \ldots, \rho_{n-1} \Gamma_{q}(\alpha)\right\} .
$$

We have the following two theorems.

Theorem 1. Assume that $\left(H_{1}\right),\left(H_{2}\right)$, and $\left(H_{4}\right)$ hold. There exists a positive $R>0$ such that problem (1) has two positive solutions $u^{*}$ and $v^{*}$ satisfying $0 \leq\left\|u^{*}\right\| \leq R$ and $0 \leq\left\|v^{*}\right\| \leq R$. Moreover, $\lim _{m \rightarrow \infty} u_{m}=u^{*}$ and $\lim _{m \rightarrow \infty} v_{m}=v^{*}, u_{m}$ and $v_{m}$ can be obtained by the following monotone iterative schemes:

$$
\begin{gathered}
u_{m}=\mathscr{T} u_{m-1}, \text { with } u_{0}(t)=R t^{\alpha-1}, \quad m=1,2, \ldots, \\
v_{m}=\mathscr{T} v_{m-1}, \text { with } v_{0}(t)=0, \quad m=1,2, \ldots
\end{gathered}
$$

In addition, we have

$$
\begin{aligned}
v_{0}(t) \leq & v_{1}(t) \leq \cdots \leq v_{m}(t) \leq \cdots \leq v^{*}(t) \leq \cdots \leq u^{*}(t) \leq \cdots \leq u_{m}(t) \leq \cdots \leq u_{2}(t) \leq u_{1}(t), \\
& D_{q}^{\alpha-i} v_{0}(t) \leq D_{q}^{\alpha-i} v_{1}(t) \leq \cdots, \leq D_{q}^{\alpha-i} v_{m}(t) \leq \cdots \leq D_{q}^{\alpha-i} v^{*}(t) \leq \cdots \leq D_{q}^{\alpha-i} u^{*}(t) \\
\leq & \cdots \leq D_{q}^{\alpha-i} u_{m}(t) \leq \cdots \leq D_{q}^{\alpha-i} u_{2}(t) \leq D_{q}^{\alpha-i} u_{1}(t), \quad i=2,3, \ldots, n-1 .
\end{aligned}
$$

Proof. First, Lemma 11 implies that $\mathscr{T} P \subset P$ for any $u \in P$ and $t \in J$. Next, let $0 \leq \lambda_{k}<1, k=1,2, \ldots, n$. For the case $\lambda_{k} \geq 1$, the proof is similar to that of $0 \leq \lambda_{k}<1$. Define

$P_{R}=\{u \in P \mid\|u\| \leq R\}$, where $R$ satisfies

$$
R \geq \max \left\{(n+1) L a_{0},\left((n+1) L a_{1}\right)^{\left(1 / 1-\lambda_{1}\right)}, \ldots,\left((n+1) L a_{n}\right)^{\left(1 / 1-\lambda_{1}\right)}\right\}
$$


For any $u \in P_{R}$, similar to (54)-(56), we obtain

$$
\begin{aligned}
&\left\|\frac{\mathscr{T} u}{1+t^{\alpha-1}}\right\|_{\infty} \leq \frac{1}{\Gamma_{q}(\alpha)-\Lambda}\left(a_{0}+\sum_{k=1}^{n} a_{k} R^{\lambda_{k}}\right)=\rho_{1}\left(a_{0}+\sum_{k=1}^{n} a_{k} R^{\lambda_{k}}\right) \leq L\left(a_{0}+\sum_{k=1}^{n} a_{k} R^{\lambda_{k}}\right) \leq R, \\
&\left\|\frac{D_{q}^{\alpha-i} \mathscr{T} u}{1+t^{i-1}}\right\|_{\infty} \leq \rho_{i} \Gamma_{q}(\alpha)\left(a_{0}+\sum_{k=1}^{n} a_{k} R^{\lambda_{k}}\right), \quad i=2,3, \ldots, n-1 \leq L\left(a_{0}+\sum_{k=1}^{n} a_{k} R^{\lambda_{k}}\right) \leq R, \quad i=2,3, \ldots, n-1, \\
&\left\|D_{q}^{\alpha-1} \mathscr{T} u\right\|_{\infty} \leq \frac{\Gamma_{q}(\alpha)}{\Gamma_{q}(\alpha)-\Lambda}\left(a_{0}+\sum_{k=1}^{n} a_{k} R^{\lambda_{k}}\right)=\rho_{1} \Gamma_{q}(\alpha)\left(a_{0}+\sum_{k=1}^{n} a_{k} R^{\lambda_{k}}\right) \leq L\left(a_{0}+\sum_{k=1}^{n} a_{k} R^{\lambda_{k}}\right) \leq R .
\end{aligned}
$$

Thus,

$$
\|\mathscr{T} u\|=\max \left\{\left\|\frac{\mathscr{T} u}{1+t^{\alpha-1}}\right\|_{\infty},\left\|\frac{D_{q}^{\alpha-i} \mathscr{T} u}{1+t^{i-1}}\right\|_{\infty},\left\|D_{q}^{\alpha-1} \mathscr{T} u\right\|_{\infty}, \quad i=2,3, \ldots, n-1\right\} \leq R
$$

That is $\mathscr{T} P_{R} \subset P_{R}$.

Denote that $u_{0}(t)=R t^{\alpha-1}$, then $u_{0}(t) \in P_{R}$, and $u_{1}(t)=\mathscr{T} u_{0}(t)$, i.e.,

$$
\begin{aligned}
u_{1}(t) & =\mathscr{T} u_{0}(t)=\int_{0}^{+\infty} G(t, q s) f\left(s, u_{0}(s), D_{q}^{\alpha-1} u_{0}(s), \ldots, D_{q}^{\alpha-(n-1)} u_{0}(s)\right) \mathrm{d}_{q} s \\
& \leq \frac{t^{\alpha-1}}{\Gamma_{q}(\alpha)-\Lambda}\left(a_{0}+\sum_{k=1}^{n} a_{k}\|u\|^{\lambda_{k}}\right) \leq t^{\alpha-1} L\left(a_{0}+\sum_{k=1}^{n} a_{k} R^{\lambda_{k}}\right) \leq R t^{\alpha-1}=u_{0}(t) .
\end{aligned}
$$

On the other hand, $\left(\mathrm{H}_{4}\right)$ implies that the operator $\mathscr{T}$ is increasing, and then we have

$$
u_{2}(t)=\mathscr{T} u_{1}(t) \leq T u_{0}(t)=u_{1}(t), \quad \forall t \in J .
$$

By the induction, we have $u_{m}(t)=\mathscr{T} u_{m}(t), m=0,1,2, \ldots$. Then, the sequence $\left\{u_{m}\right\}_{m=0}^{\infty} \subset \mathscr{T}\left(\Omega_{R}\right) \subset P_{R}$ and satisfies the following relation: 
$u_{m+1}(t) \leq u_{m}(t), \quad \forall t \in J, m=0,1,2, \ldots$
By Lemmas 5 and 7, we have

$$
\begin{aligned}
D_{q}^{\alpha-i} u_{1}(t) & =D_{q}^{\alpha-i} \mathscr{T} u_{0}(t)=\int_{0}^{+\infty} G_{i}^{*}(t, q s) f\left(s, u_{0}(s), D_{q}^{\alpha-1} u_{0}(s), \ldots, D_{q}^{\alpha-(n-1)} u_{0}(s)\right) \mathrm{d}_{q} s \\
& \leq \frac{\Gamma_{q}(\alpha) t^{i-1}}{\Gamma_{q}(i)\left(\Gamma_{q}(\alpha)-\Lambda\right)} f\left(s, u_{0}(s), D_{q}^{\alpha-1} u_{0}(s), \ldots, D_{q}^{\alpha-(n-1)} u_{0}(s)\right) \mathrm{d}_{q} s \\
& =\frac{1}{\Gamma_{q}(\alpha)-\Lambda} D_{q}^{\alpha-i} t^{\alpha-1} f\left(s, u_{0}(s), D_{q}^{\alpha-1} u_{0}(s), \ldots, D_{q}^{\alpha-(n-1)} u_{0}(s)\right) \mathrm{d}_{q} s \\
& \leq \frac{1}{\Gamma_{q}(\alpha)-\Lambda}\left(a_{0}+\sum_{k=1}^{n} a_{k} R^{\lambda_{k}}\right) D_{q}^{\alpha-i} t^{\alpha-1} \leq L\left(a_{0}+\sum_{k=1}^{n} a_{k} R^{\lambda_{k}}\right) D_{q}^{\alpha-i} t^{\alpha-1} \\
& \leq \mathrm{RD}_{q}^{\alpha-i} t^{\alpha-1}=D_{q}^{\alpha-i} u_{0}(t), \quad i=1,2, \ldots, n-1, \\
D_{q}^{\alpha-i} u_{2}(t) & =D_{q}^{\alpha-i} \mathscr{T} u_{1}(t) \leq D_{q}^{\alpha-i} \mathscr{T} u_{0}(t)=D_{q}^{\alpha-i} u_{1}(t), \quad i=1,2, \ldots, n-1 .
\end{aligned}
$$

By the induction, we have

$$
D_{q}^{\alpha-i} u_{m+1}(t) \leq D_{q}^{\alpha-i} u_{m}(t), \quad \forall t \in J, i=1,2, \ldots, n-1, m=0,1,2, \ldots
$$

In view of the complete continuity of the operator $\mathscr{T}$ and $u_{m+1}(t)=\mathscr{T} u_{m}(t),\left\{u_{m}(t)\right\}_{m=0}^{\infty}$ is relatively compact. That is, $\left\{u_{m}(t)\right\}_{m=0}^{\infty}$ has a convergent subsequence $\left\{u_{m_{k}}(t)\right\}_{k=1}^{\infty}$ and there exists a $u^{*} \in P_{R}$ such that $u_{m_{k}} \longrightarrow u^{*}$ as $k \longrightarrow \infty$. This together with (90) implies $\lim _{m \longrightarrow \infty} u_{m}=u^{*}$. Since $\mathscr{T}$ is continuous and $u_{m+1}(t)=\mathscr{T} u_{m}(t)$, then we have $u^{*}=\mathscr{T} u^{*}$, that is, $u^{*}$ is a fixed point of operator $\mathscr{T}$.

For the scheme $\left\{v_{m}\right\}_{m=0}^{\infty}$, we use a similar discussion. For $t \in J$, by Lemma 5 and $f \in C\left(J \times \mathbb{R}^{n}, J\right)$, we have

$$
v_{1}(t)=\mathscr{T} v_{0}=\int_{0}^{+\infty} G(t, q s) f\left(s, v_{0}(s), D_{q}^{\alpha-1} v_{0}(s) \mathrm{d}_{q} s, \ldots, D_{q}^{\alpha-(n-1)} v_{0}(s)\right) \mathrm{d}_{q} s \geq 0=v_{0}(t)
$$

Applying the monotonicity of function $f$, we have

$$
v_{m+1}(t)=\mathscr{T} v_{m}(t) \text { and } v_{m}(t) \leq v_{m+1}(t), \quad m=0,1,2, \ldots
$$

$$
v_{2}(t)=\mathscr{T} v_{1}(t) \geq \mathscr{T} v_{0}(t)=v_{1}(t), \quad \forall t \in J .
$$

By the induction, we have

By Remark 2 and Lemma 6, we get

$$
D_{q}^{\alpha-i} v_{1}(t)=D_{q}^{\alpha-i} \mathscr{T} v_{0}(t)=\int_{0}^{+\infty} G_{i}^{*}(t, q s) f\left(s, v_{0}(s), D_{q}^{\alpha-1} v_{0}(s) \mathrm{d}_{q} s\right) \geq 0=D_{q}^{\alpha-i} v_{0}(t), \quad i=1,2, \ldots, n-1
$$

By the induction, we have

$$
D_{q}^{\alpha-i} v_{m}(t) \leq D_{q}^{\alpha-i} v_{m+1}(t), \quad i=1,2, \ldots, n-1, m=0,1,2, \ldots
$$

Using the complete continuity of the operator $\mathscr{T}$ and the iterative scheme, it is easy to get

$$
v_{m} \longrightarrow v^{*} \text { and } \mathscr{T} v^{*}=v^{*}
$$

Finally, we show that $u^{*}$ and $v^{*}$ are the maximal and minimal positive solutions of problem (1). Let $w(t)$ be any positive solution of (1), then

$$
\begin{aligned}
v_{0}(t) & =0<w(t) \leq R t^{\alpha-1}=u_{0}(t), \\
D_{q}^{\alpha-i} v_{0}(t) & \leq D_{q}^{\alpha-i} w(t) \leq D_{q}^{\alpha-i} u_{0}(t) .
\end{aligned}
$$

Noting that $\mathscr{T}$ is increasing, then we have 
$v_{1}(t)=\mathscr{T} v_{0}(t)<\mathscr{T} w(t)=w(t) \leq \mathscr{T} u_{0}(t)=u_{1}(t), \quad$ for all $t \in J$.

(100)
Since $v^{*}=\lim _{m \longrightarrow \infty} v_{m}$ and $u^{*}=u_{m_{m \rightarrow \infty}} \lim$, it follows that, for $t \in J$ and $i=1,2, \ldots, n-1$, we have

Similarly, for $t \in J$ and $i=1,2, \ldots, n-1$, we can get

$$
\begin{aligned}
v_{m}(t) & \leq w(t) \leq u_{m}(t) \\
D_{q}^{\alpha-i} v_{m}(t) & \leq D_{q}^{\alpha-i} w(t) \leq D_{q}^{\alpha-i} u_{m}(t) .
\end{aligned}
$$

$$
\begin{aligned}
v_{0}(t) & \leq v_{1}(t) \leq \cdots \leq v_{m}(t) \leq \cdots \leq v^{*} \leq w(t) \leq u^{*} \leq \cdots \leq u_{1}(t) \leq u_{0}(t), \\
D_{q}^{\alpha-i} v_{0}(t) & \leq D_{q}^{\alpha-i} v_{1}(t) \leq \cdots \leq D_{q}^{\alpha-i} v_{m}(t) \leq \cdots \leq D_{q}^{\alpha-i} v^{*}(t) \\
& \leq D_{q}^{\alpha-i} w(t) \leq D_{q}^{\alpha-i} u^{*}(t) \leq \cdots \leq D_{q}^{\alpha-i} u_{1}(t) \leq D_{q}^{\alpha-i} u_{0}(t) .
\end{aligned}
$$

This implies that $v^{*}(t)$ and $u^{*}(t)$ are the minimal and maximal solutions of problem (1), and (53) and (54) hold.

On the other hand, we infer that $v^{*}(t)$ and $u^{*}(t)$ are two positive solutions of (1). In fact, by the condition $\left(H_{4}\right)$, we get 0 function is not the solution of problem (1), which means $v^{*}(t)>0, u^{*}(t)>0$, and they can be constructed by means of two monotone iterative schemes in (81) and (82).

Theorem 2. Suppose conditions $\left(H_{1}\right)$ and $\left(H_{3}\right)$ are satisfied. If

$$
A:=L \sum_{k=1}^{n} b_{k}<1,
$$

then problem (1) has a unique positive solution $\widetilde{u}(t)$ in $P$. Moreover, there is an iterative scheme $\left\{u_{m}\right\}_{m=0}^{\infty}$ such that $u_{m} \longrightarrow \widetilde{u}$ as $m \longrightarrow \infty$ uniformly on any finite interval of $J$, where

$$
u_{m}=\mathscr{T} u_{m-1}, \quad m=1,2, \ldots,
$$

hold. $L$ is defined in (80). In addition, there is an error estimate for the approximation scheme

$$
\left\|u_{m}-\tilde{u}\right\|=\frac{A^{m-1}}{1-A}\left\|u_{1}-u_{0}\right\|, \quad m=1,2, \ldots
$$

Proof. Choose $r>(L \kappa / 1-A)$, where $\kappa$ is defined in $\left(H_{3}\right)$. Let $P_{r}=\{u \in P \mid\|u\| \leq r\}$. For any $u \in P_{r}$, by Lemmas 5-7 and 10, we have

$$
\begin{aligned}
& \left\|\frac{\mathscr{T} u}{1+t^{\alpha-1}}\right\|_{\infty} \leq L\left(\sum_{k=1}^{n} b_{k} r+\kappa\right)<A r+L \kappa, \quad \forall u \in P_{r}, \\
& \left\|\frac{D_{q}^{\alpha-i} \mathscr{T} u}{1+t^{i-1}}\right\|_{\infty} \leq L\left(\sum_{k=1}^{n} b_{k} r+\kappa\right)<A r+L \kappa, \quad \forall u \in P_{r}, i=2,3, \ldots, \\
& \left\|D_{q}^{\alpha-1} \mathscr{T} u\right\|_{\infty} \leq L\left(\sum_{k=1}^{n} b_{k} r+\kappa\right)<A r+L \kappa, \quad \forall u \in P_{r} .
\end{aligned}
$$

Thus, we have

$\|\mathscr{T} u\| \leq A r+L \kappa<A r+(1-A) r=r, \quad \forall u \in P_{r}$, i.e., $\mathscr{T} P_{r} \subset P_{r}$.

In the following, we show that $\mathscr{T}$ is a contraction. For any $u_{1}, u_{2} \in P_{r}$, by condition $\left(H_{3}\right)$ and Lemma 10 , we obtain 


$$
\begin{aligned}
\left\|\frac{\mathscr{T} u_{2}}{1+t^{\alpha-1}}-\frac{\mathscr{T} u_{1}}{1+t^{\alpha-1}}\right\|_{\infty}= & \sup _{t \in J}\left|\int_{0}^{+\infty} \frac{G(t, q s)}{1+t^{\alpha-1}}\left(\begin{array}{c}
f\left(s, u_{2}(s), D_{q}^{\alpha-1} u_{2}(s), \ldots, D_{q}^{\alpha-(n-1)} u_{2}(s)\right) \\
-f\left(s, u_{1}(s), D_{q}^{\alpha-1} u_{1}(s), \ldots, D_{q}^{\alpha-(n-1)} u_{1}(s)\right)
\end{array}\right)\right| \mathrm{d}_{q} s \\
\leq & L \int_{0}^{+\infty}\left(b_{1}(s)\left(1+s^{\alpha-1}\right) \frac{\left|u_{2}(s)-u_{1}(s)\right|}{1+s^{\alpha-1}}+b_{2}(s)\left|D_{q}^{\alpha-1} u_{2}(s)-D_{q}^{\alpha-1} u_{1}(s)\right|\right. \\
& +b_{3}(s)(1+s) \frac{\left|D_{q}^{\alpha-2} u_{2}(s)-D_{q}^{\alpha-2} u_{1}(s)\right|}{1+s}+\cdots+b_{i+1}(s)\left(1+s^{i-1}\right) \frac{\left|D_{q}^{\alpha-i} u_{2}(s)-D_{q}^{\alpha-i} u_{1}(s)\right|}{1+s^{i-1}}+\cdots \\
& \left.+b_{n}(s)\left(1+s^{n-2}\right) \frac{\left|D_{q}^{\alpha-(n-1)} u_{2}(s)-D_{q}^{\alpha-(n-1)} u_{1}(s)\right|}{1+s^{n-2}}\right) \mathrm{d}_{q} s \\
\leq & L\left(b_{1}\left\|\frac{u_{2}-u_{1}}{1+t \alpha-1}\right\|_{\infty}+b_{2}\left\|D_{q}^{\alpha-1} u_{2}-D_{q}^{\alpha-1} u_{1}\right\|_{\infty}+\cdots+b_{n}\left\|D_{q}^{\alpha-(n-1)} u_{2}-D_{q}^{\alpha-(n-1)} u_{1}\right\|_{\infty}\right) \\
\leq & L \sum_{k=1}^{n} b_{k}\left\|u_{2}-u_{1}\right\|_{\infty}
\end{aligned}
$$

In the same way, we have

$$
\begin{gathered}
\left\|D_{q}^{\alpha-i} \frac{\mathscr{T} u_{2}}{1+t^{i-1}}-\frac{D_{q}^{\alpha-i} \mathscr{T} u_{1}}{1+t^{i-1}}\right\|_{\infty} \leq L \sum_{k=1}^{n} b_{k}\left\|u_{2}-u_{1}\right\|_{\infty}, \quad i=2,3, \ldots, n-1, \\
\left\|D_{q}^{\alpha-i} u_{2}-D_{q}^{\alpha-1} u_{1}\right\| \leq L \sum_{k=1}^{n} b_{k}\left\|u_{2}-u_{1}\right\|_{\infty} .
\end{gathered}
$$

From (108)-(110), we have $\left\|\mathscr{T} u_{2}-\mathscr{T} u_{1}\right\| \leq L \sum_{k=1}^{n} b_{k}\left\|u_{2}-u_{1}\right\|=A\left\|u_{2}-u_{1}\right\|, \quad \forall u_{1}, u_{2} \in P_{r}$.
Since $A<1$, then $\mathscr{T}$ is a contraction. The Banach fixed point theorem ensures that $\mathscr{T}$ has a unique fixed point $\widetilde{u} \in P$. Hence, problem (1) has a unique positive solution $\tilde{u}$.

Moreover, for any $u_{0} \in P,\left\{u_{m}\right\}_{m=0}^{\infty},\left\|u_{m}-\tilde{u}\right\| \longrightarrow 0$ as $m \longrightarrow 0$, where $u_{m}=\mathscr{T} u_{m-1}$. By (111), we have

$$
\begin{aligned}
\left\|u_{m}-u_{m-1}\right\| & \leq A\left\|u_{m-1}-u_{m-2}\right\| \leq \cdots \leq A^{m-1}\left\|u_{1}-u_{0}\right\| \\
\left\|u_{m}-u_{l}\right\| & \leq\left\|u_{m}-u_{m-1}\right\|+\left\|u_{m-1}-u_{m-2}\right\|+\cdots+\left\|u_{l+1}-u_{l}\right\| \\
& \leq A^{m-1}\left\|u_{1}-u_{0}\right\|+A^{m-2}\left\|u_{1}-u_{0}\right\|+\cdots+A^{l}\left\|u_{1}-u_{0}\right\|=\frac{A^{m-1}\left(1-A^{m-l}\right)}{1-A}\left\|u_{1}-u_{0}\right\| .
\end{aligned}
$$

Let $l \longrightarrow \infty$ on both sides of (113), we obtain

$$
\left\|u_{m}-\tilde{u}\right\| \leq \frac{A^{m-1}}{1-A}\left\|u_{1}-u_{0}\right\|
$$

Remark 1. The function $f$ in problem (1) relies on lowerorder fractional $q$-derivative of unknown function which is different from [17].
Remark 2. The results in Theorems 1 and 2 extend the fractional $q$-derivative from the low-order to the high-order fractional derivative.

Remark 3. The two iterative sequences in Theorem 1 begin with some simple functions which are helpful for computational purpose. 


\section{Example}

Consider the following equation:

$$
\left\{\begin{array}{l}
D_{q}^{(5 / 2)} u(t)+f\left(t, u(t), D_{q}^{(3 / 2)} u(t), D_{q}^{(1 / 2)} u(t)\right)=0 \\
u(0)=D_{q} u(0)=0 \\
D_{q}^{(3 / 2)} u(+\infty)=\int_{0}^{+\infty} e^{-t} t^{-(3 / 2)} u(t) \mathrm{d}_{q} t
\end{array}\right.
$$

where $\quad n=3, \alpha=(5 / 2), q=(1 / 2), \quad g(t)=e^{-t} t^{-(3 / 2)}, \lambda_{1}$ $=0.1, \lambda_{2}=0.2, \lambda_{3}=0.4$, and function

$$
f\left(t, u(t), D_{q}^{(3 / 2)} u(t), D_{q}^{(1 / 2)} u(t)\right)=\frac{4}{(5+t)^{2}}+\frac{e^{-t}|u(t)|^{0.1}}{\left(1+t^{(3 / 2)}\right)^{0.1}}+\frac{2 t\left|D_{q}^{(3 / 2)} u\right|^{0.2}}{\left(5+t^{2}\right)^{2}}+\frac{e^{-2 t}\left|D_{q}^{(1 / 2)} u(t)\right|^{0.4}}{(1+t)^{0.4}}
$$

By computation, we deduce that

$$
\begin{aligned}
\left|f\left(t, u(t), D_{q}^{(3 / 2)} u(t), D_{q}^{(1 / 2)} u(t)\right)\right| & \leq \frac{4}{(5+t)^{2}}+\frac{e^{-t}|u(t)|^{0.1}}{\left(1+t^{(3 / 2)}\right)^{0.1}}+\frac{2 t\left|D_{q}^{(3 / 2)} u\right|^{0.2}}{\left(5+t^{2}\right)^{2}}+\frac{e^{-2 t}\left|D_{q}^{(1 / 2)} u(t)\right|^{0.4}}{(1+t)^{0.4}} \\
& =a_{0}(t)+a_{1}(t)|u(t)|^{0.1}+a_{2}(t)\left|D_{q}^{(3 / 2)} u(t)\right|^{0.2}+a_{3}(t)\left|D_{q}^{(1 / 2)} u(t)\right|^{0.4} \\
a_{0} & =\int_{0}^{+\infty} a_{0}(t) \mathrm{d}_{q} t=\int_{0}^{+\infty} \frac{4}{(5+t)^{2}} \mathrm{~d}_{q} t<\frac{1}{12}, \\
a_{1} & =\int_{0}^{+\infty} a_{1}(t)\left(1+t^{(3 / 2)}\right)^{0.1} \mathrm{~d}_{q} t=\int_{0}^{+\infty} \frac{e^{-t}}{\left(1+t^{(3 / 2)}\right)^{0.1}}\left(1+t^{(3 / 2)}\right)^{0.1} \mathrm{~d}_{q} t<\int_{0}^{+\infty} \frac{1}{e^{t}} \mathrm{~d}_{q} t<\frac{1}{e}, \\
a_{2} & =\int_{0}^{+\infty} a_{2}(t) \mathrm{d}_{q} t=\int_{0}^{+\infty} \frac{2 t}{\left(5+t^{2}\right)^{2}} \mathrm{~d}_{q} t<\frac{1}{24}, \\
a_{3} & =\int_{0}^{+\infty} a_{1}(t)(1+t)^{0.4} \mathrm{~d}_{q} t=\int_{0}^{+\infty} \frac{e^{-2 t}}{\left((1+t)^{0.4}\right)^{0.4}}\left((1+t)^{0.4}\right)^{0.4} \mathrm{~d}_{q} t<\int_{0}^{+\infty} \frac{1}{e^{2 t}} \mathrm{~d}_{q} t<\frac{1}{2 e},
\end{aligned}
$$

which means that hypothesis $\left(H_{2}\right)$ is satisfied.

On the other hand, $\Lambda=\int_{0}^{+\infty} t^{(3 / 2)} g(t) \mathrm{d}_{q} t=\int_{0}^{+\infty}$ $t^{(3 / 2)} e^{-t} t^{(3 / 2)} \mathrm{d}_{q} t=\int_{0}^{+\infty} e^{-t} \mathrm{~d}_{q} t<(1 / e)<\Gamma_{(1 / 2)}(5 / 2), \quad$ and $f(t, 0,0,0) \equiv 0$. So condition $\left(H_{1}\right)$ is satisfied. Moreover, from the expression of the function $f$, it is obvious that $f$ is increasing with respect to the variables $u_{1}, u_{2}, u_{3}, \forall t \in J$. Hence, condition $\left(\mathrm{H}_{4}\right)$ is satisfied. By Theorem 1, it follows that problem (1) has two positive solutions, which can be given by the limits of two explicit monotone iterative schemes in (81) and (82).

\section{Conclusions}

In this paper, we study the boundary value problem of a fractional $q$-difference equation with nonlocal integral boundary conditions on the half-line. We obtain some new results as follows: (1) the existence and uniqueness of positive solutions for a higher-order nonlinear fractional $q$-difference equation with integral boundary conditions on the half-line are obtained, and (2) the monotone iterative schemes of positive solutions for the problem which considered in our paper are obtained.

\section{Data Availability}

All the data included in this study are available upon request by contact with the corresponding author.

\section{Conflicts of Interest}

The authors declare no conflicts of interest.

\section{Acknowledgments}

This research was supported by the Science and Technology Foundation of Guizhou Province (Grant nos. [2016]7075 
and [2019]1162), the Project for Young Talents Growth of Guizhou Provincial Department of Education under (Grant no. Ky[2017]133), and the Project of Guizhou Minzu University under (Grant no. 16yjrcxm002).

\section{References}

[1] F. H. Jackson, "On $q$-functions and a certain difference operator," Transactions of the Royal Society of Edinburgh Earth Sciences, vol. 46, pp. 253-281, 1908.

[2] F. H. Jackson, "On $q$-definite integrals," The Quarterly journal of pure and applied mathematics, vol. 41, pp. 193-203, 1910.

[3] W. A. Al-Salam, "Some fractional $q$-integral and $q$-derivatives," Proceedings of the Edinburgh Mathematical Society, vol. 15, pp. 135-140, 1916.

[4] R. P. Agarwal, "Certain fractional q-integrals and q-derivatives," Mathematical Proceedings of the Cambridge Philosophical Society, vol. 66, no. 2, pp. 365-370, 1969.

[5] R. A. C. Ferreira, "Positive solutions for a class of boundary value problems with fractionalq-differences," Computers \& Mathematics with Applications, vol. 61, no. 2, pp. 367-373, 2011.

[6] J. R. Graef and L. Kong, "Positive solutions for a class of higher order boundary value problems with fractional q-derivatives," Applied Mathematics and Computation, vol. 218, no. 19, pp. 9682-9689, 2012.

[7] L. Yang, H. Chen, L. Luo, and Z. Luo, "Successive iteration and positive solutions for boundary value problem of nonlinear fractional $q$-difference equation," Journal of Applied Mathematics and Computing, vol. 42, no. 1-2, pp. 89-102, 2013.

[8] J. Ma and J. Yang, "Existence of solutions for multi-point boundary value problem of fractional $q$-difference equation," Electronic Journal of Qualitative Theory of Differential Equations, vol. 92, no. 92, pp. 1-10, 2011.

[9] T. Abdeljawad and D. Baleanu, "Caputo $q$-fractional initial value problems and a q-analogue Mittag-Leffler function," Communications in Nonlinear Science and Numerical Simulation, vol. 16, no. 12, pp. 4682-4688, 2011.

[10] B. Ahmad, S. K. Ntouyas, and I. K. Purnaras, "Existence results for nonlocal boundary value problems of nonlinear fractional q-difference equations," Advances in Difference Equations, vol. 2012, no. 1, Article ID 140, 2012.

[11] N. Khodabakhshi and S. M. Vaezpour, "Existence and uniqueness of positive solution for a class of boundary value problems with fractional-differences," Journal of Nonlinear and Convex Analysis, vol. 16, pp. 375-384, 2015.

[12] X. Li, Z. Han, and X. Li, "Boundary value problems of fractional q-difference Schröinger equations," Applied Mathematics Letters, vol. 46, pp. 100-105, 2015.

[13] G. Wang, "Twin iterative positive solutions of fractional $q$ difference Schrödinger equations," Applied Mathematics Letters, vol. 76, pp. 103-109, 2018.

[14] G. Wang, Z. Bai, Z. Bai, and L. Zhang, "Successive iterations for unique positive solution of a nonlinear fractional $Q$-integral boundary value problem," Journal of Applied Analysis \& Computation, vol. 9, no. 4, pp. 1204-1215, 2019.

[15] A. Ricardo and M. Natália, "Existence results for fractional $q$ difference equations of $\alpha \in(2,3)$ order with three-point boundary conditions," Communications in Nonlinear Science and Numerical Simulationl, vol. 19, pp. 1675-1685, 2019.

[16] Y. Zhao, H. Chen, and Q. Zhang, "Existence results for fractional-difference equations with nonlocal-integral boundary conditions," Advances in Difference Equations, vol. 48, 2013.

[17] K. K. Ma, X. H. Li, and S. R. Sun, "Boundary value problems of fractionalq-difference equations on the half-line," 2019.

[18] Y. Li, J. Liu, D. O'Regan, and J. Xu, "Nontrivial solutions for a system of fractional $q$-difference equations involving q-integral boundary conditions," Mathematics, vol. 8 , no. 5, p. 828, 2020.

[19] J. Mao, Z. Zhao, and C. Wang, "The unique iterative positive solution of fractional boundary value problem with $q$-difference," Applied Mathematics Letters, vol. 100, Article ID 106002, 2020.

[20] J. Ren and C. Zhai, "Unique solutions for fractional $q$-difference boundary value problems via a fixed point method," Bulletin of the Malaysian Mathematical Sciences Society, vol. 42, no. 4, pp. 1507-1521, 2019.

[21] W. Yang, "Positive solutions for nonlinear semipositone fractional $q$-difference system with coupled integral boundary conditions," Applied Mathematics and Computation, vol. 244, pp. 702-725, 2014.

[22] F. Guo and S. Kang, "Positive solutions for a class of fractional boundary value problem with $q$-derivatives," Mediterranean Journal of Mathematic, vol. 16, p. 113, 2019.

[23] V. Kac and P. Cheung, Quantum Calculus, Springer, Berlin, Germany, 2002.

[24] P. M. Rajkovi, S. D. Marinkovi, and M. S. Stankovi, "Fractional integrals and derivatives in q-calculus," Applicable Analysis and Discrete Mathematics (AADM), vol. 1, pp. 311323, 2007.

[25] R. Ferreira, "Nontrivial solutions for fractionalq-difference boundary value problems," Electronic Journal of Qualitative Theory of Differential Equations, vol. 70, no. 70, pp. 1-10, 2010.

[26] X. Su and S. Zhang, "Unbounded solutions to a boundary value problem of fractional order on the half-line," Computers \& Mathematics with Applications, vol. 61, no. 4, pp. 10791087, 2011. 